\title{
HERSCHEL/PACS SPECTROSCOPIC SURVEY OF PROTOSTARS IN ORION: THE ORIGIN OF FAR-INFRARED CO EMISSION*
}

\author{
P. Manoj ${ }^{1}$, D. M. Watson ${ }^{1}$, D. A. Neufeld ${ }^{2}$, S. T. Megeath ${ }^{3}$, R. Vavrek ${ }^{4}$, Vincent Yu ${ }^{1}$, R. Visser $^{5}$,

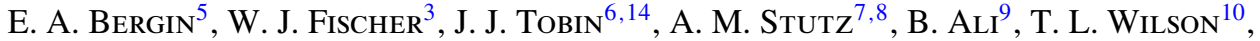 \\ J. Di Francesco ${ }^{11}$, M. Osorio ${ }^{12}$, S. Maret ${ }^{13}$, and C. A. Poteet ${ }^{3}$ \\ ${ }^{1}$ Department of Physics and Astronomy, University of Rochester, Rochester, NY 14627, USA; manoj@ pas.rochester.edu \\ 2 Department of Physics and Astronomy, Johns Hopkins University, 3400 North Charles Street, Baltimore, MD 21218, USA \\ ${ }^{3}$ Department of Physics and Astronomy, University of Toledo, 2801 West Bancroft Street, OH 43606, USA \\ ${ }^{4}$ European Space Agency, ESAC/SRE-OAH, P.O. Box 78, E-28691 Villanueva de la Cañada, Madrid, Spain \\ ${ }^{5}$ Department of Astronomy, University of Michigan, 500 Church Street, Ann Arbor, MI 48109, USA \\ ${ }^{6}$ National Radio Astronomy Observatory, Charlottesville, VA 22903, USA \\ ${ }^{7}$ Max-Planck-Institut für Astronomie, Königstuhl 17, D-69117 Heidelberg, Germany \\ ${ }^{8}$ Steward Observatory, University of Arizona, 933 North Cherry Avenue, Tucson, AZ 85721, USA \\ ${ }^{9}$ NHSC/IPAC/Caltech, 770 South Wilson Avenue, Pasadena, CA 91125, USA \\ ${ }^{10}$ US Naval Research Laboratory, Code 7210, Washington, DC 20375, USA \\ ${ }^{11}$ National Research Council of Canada, Herzberg Institute of Astrophysics, Department of Physics and Astronomy, \\ University of Victoria, Victoria, BC V9E 2E7, Canada \\ 12 Instituto de Astrofisica de Andalucia, CSIC, Camino Bajo de Húetor 50, E-18008 Granada, Spain \\ ${ }^{13}$ Laboratoire d'Astrophysique de Grenoble, Observatoire de Grenoble, Université Joseph Fourier, CNRS, UMR 571, F-38041 Grenoble, France \\ Received 2012 June 8; accepted 2012 November 9; published 2013 January 11
}

\begin{abstract}
We present far-infrared $(57-196 \mu \mathrm{m})$ spectra of 21 protostars in the Orion molecular clouds. These were obtained with the Photodetector Array Camera and Spectrometer (PACS) on board the Herschel Space observatory as part of the Herschel Orion Protostar Survey program. We analyzed the emission lines from rotational transitions of CO, involving rotational quantum numbers in the range $J_{\text {up }}=14-46$, using PACS spectra extracted within a projected distance of $\lesssim 2000$ AU centered on the protostar. The total luminosity of the CO lines observed with PACS $\left(L_{\mathrm{CO}}\right)$ is found to increase with increasing protostellar luminosity $\left(L_{\mathrm{bol}}\right)$. However, no significant correlation is found between $L_{\mathrm{CO}}$ and evolutionary indicators or envelope properties of the protostars such as bolometric temperature, $T_{\text {bol }}$, or envelope density. The CO rotational (excitation) temperature implied by the line ratios increases with increasing rotational quantum number $J$, and at least 3-4 rotational temperature components are required to fit the observed rotational diagram in the PACS wavelength range. The rotational temperature components are remarkably invariant between protostars and show no dependence on $L_{\mathrm{bol}}, T_{\mathrm{bol}}$, or envelope density, implying that if the emitting gas is in local thermodynamic equilibrium, the $\mathrm{CO}$ emission must arise in multiple temperature components that remain independent of $L_{\text {bol }}$ over two orders of magnitudes. The observed CO emission can also be modeled as arising from a single-temperature gas component or from a medium with a power-law temperature distribution; both of these require sub-thermally excited molecular gas at low densities $\left(n\left(\mathrm{H}_{2}\right) \lesssim 10^{6} \mathrm{~cm}^{-3}\right)$ and high temperatures $(T \gtrsim 2000 \mathrm{~K}$ ). Our results suggest that the contribution from photodissociation regions, produced along the envelope cavity walls from UV-heating, is unlikely to be the dominant component of the CO emission observed with PACS. Instead, the "universality" of the rotational temperatures and the observed correlation between $L_{\mathrm{CO}}$ and $L_{\text {bol }}$ can most easily be explained if the observed $\mathrm{CO}$ emission originates in shock-heated, hot ( $T \gtrsim 2000 \mathrm{~K})$, sub-thermally excited $\left(n\left(\mathrm{H}_{2}\right) \lesssim 10^{6} \mathrm{~cm}^{-3}\right)$ molecular gas. Post-shock gas at these densities is more likely to be found within the outflow cavities along the molecular outflow or along the cavity walls at radii $\gtrsim$ several 100-1000 AU.
\end{abstract}

Key words: circumstellar matter - ISM: jets and outflows - molecular processes - stars: formation - techniques: spectroscopic

Online-only material: color figures

\section{INTRODUCTION}

The early evolution of protostars is driven by the competition between infall and outflow (Shu et al. 1987; Stahler \& Palla 2005; McKee \& Ostriker 2007; Hartmann 2009). Energetic processes associated with mass accretion and ejection heat up the surrounding gas to temperatures of several $100 \mathrm{~K}$ to several 1000 K (e.g., van Dishoeck et al. 2009). The infalling envelope material first lands on the protostellar disk before

\footnotetext{
* Herschel is an ESA space observatory with science instruments provided by European-led Principal Investigator consortia and with important participation from NASA.

${ }^{14}$ Hubble Fellow.
}

accreting onto the central star (Cassen \& Moosman 1981; Neufeld \& Hollenbach 1994; Hartmann 2009). Ultraviolet radiation produced by accretion onto a protostar can heat up the circumstellar gas to a few $100 \mathrm{~K}$ (Spaans et al. 1995; van Kempen et al. 2009a). Protostars drive powerful bipolar jets and outflows into the surrounding medium, producing shocks that compress and heat up the envelope or ambient material to several $1000 \mathrm{~K}$ even at relatively large distances ( from the protostar (Bachiller 1996; Bachiller \& Tafalla 1999; Reipurth \& Bally 2001; Hollenbach \& McKee 1989; Kaufman $\&$ Neufeld 1996). While the lowest rotational transitions $(J \leqslant 3)$ of CO have been widely used to trace cold $(T \lesssim 30 \mathrm{~K})$ molecular gas associated with protostars, the far-infrared (FIR) CO lines 
$(J \gtrsim 14)$ are better diagnostics of warm and hot molecular gas (Watson et al. 1980; Storey et al. 1981; Giannini et al. 2001; Nisini et al. 2002). These latter lines can in principle be used to characterize the density, temperature, molecular abundance, and spatial extent of the emitting region (Watson et al. 1985; Draine et al. 1983; Hollenbach \& McKee 1989; Kaufman \& Neufeld 1996). The spatial distribution and physical conditions in the emitting gas can provide vital clues about the heating mechanisms and therefore also on the various energetic processes associated with the earliest phases of star formation.

The first detection of the FIR CO lines from an astronomical source was toward Orion-KL, with the Kuiper Airborne Observatory (KAO; Watson et al. 1980). Because of low sensitivity, the KAO could only observe the brightest regions around luminous and massive protostars (Storey et al. 1981; Stacey et al. 1982, 1983; Jaffe et al. 1987). These early observations of very massive protostars suggested that FIR CO emission arises in gas heated by non-dissociative, magnetohydrodynamic shocks (Watson et al. 1985; Storey et al. 1981). FIR CO lines toward several low-mass protostars were later observed with the Infrared Space Observatory (ISO; e.g., Nisini et al. 1997; Ceccarelli et al. 1998; Nisini et al. 1999; Benedettini et al. 2000; Saraceno et al. 1999a, 1999b; Giannini et al. 2001; Nisini et al. 2002; van Dishoeck 2004). Modeling based on the ISO data found that the observed $\mathrm{CO}$ emission originates in molecular gas within a projected distance of $\lesssim 1500 \mathrm{AU}$ from the protostar, with temperatures in the range of $200-2000 \mathrm{~K}$ and $\mathrm{H}_{2}$ densities in the range of $10^{4}-10^{7} \mathrm{~cm}^{-3}$ (Ceccarelli et al. 1998; Nisini et al. 1999; Benedettini et al. 2000; Giannini et al. 2001; Nisini et al. 2002). These studies suggested slow $\left(v_{s} \lesssim 20 \mathrm{~km} \mathrm{~s}^{-1}\right)$ nondissociative shocks as the main mechanism for the heating of the molecular gas. However, based on the similarity between the observed $\mathrm{CO}$ line ratios of protostars and Herbig Ae/Be stars, Ceccarelli (2000) proposed that CO $J_{\text {up }}=14-21$ lines from protostars probably originate in the dense photodissociation regions (PDRs) created by UV photons from the central objects. A lack of spatial information (beam size $\sim 80^{\prime \prime}$ ) and low spectral resolution of the $I S O$-LWS, however, prevented any further progress in disentangling the various components contributing to the FIR CO emission.

With the Photodetector Array Camera and Spectrometer (PACS; Poglitsch et al. 2010) on board the Herschel Space Observatory (Pilbratt et al. 2010), it has now been possible to observe the rotational transitions from the entire CO ladder from $J=13-12$ to $J=49-48$ in the FIR wavelength range (van Kempen et al. 2010a, 2010b; Fich et al. 2010; Herczeg et al. 2012; Goicoechea et al. 2012) at high spatial resolution (beam size $\sim 10^{\prime \prime}$ at $63 \mu \mathrm{m}$ ) and unprecedented sensitivity. Modeling of the absolute and relative line fluxes of more than $30 \mathrm{CO}$ rotational transitions accessible with PACS can be used to place strong constraints on the density and temperature of the emitting medium (e.g., Neufeld 2012). However, the CO lines observed with PACS are spectrally unresolved (e.g., van Kempen et al. 2010a, 2010b; Herczeg et al. 2012), rendering it difficult to separate out the contribution from various velocity components of the emitting gas to the total observed line flux. Spectrally resolved observations of the lowest- $J$ CO lines $\left(J_{\text {up }} \lesssim 8\right)$ toward low-mass protostars in the submillimeter wavelengths have shown the presence of narrow $\left(\mathrm{FWHM} \lesssim 2 \mathrm{~km} \mathrm{~s}^{-1}\right.$ ) and relatively broad (FWHM $\sim 10-30 \mathrm{~km} \mathrm{~s}^{-1}$ ) velocity components in the profiles of these lines. The narrow component was found to dominate the total line flux for these transitions (van Kempen et al. 2009a, 2009b; van Dishoeck et al. 2009; Y1ldız et al.
2012). For lines with $J_{\text {up }} \lesssim 4$, these studies attributed the narrow component as due to emission from passively heated protostellar envelope; for CO $J=6-5$ and CO $J=7-6$ transitions the narrow component was found to be dominated by emission from UV-photon-heated gas. Early modeling of the unresolved CO lines observed with Herschel/PACS ( $J_{\text {up }} \gtrsim 14$ ) toward a few protostars suggested that the emission from UV-heated PDRs dominated the line flux even in the PACS range up to $J_{\text {up }}=$ 20 (van Kempen et al. 2010b). These studies have shown that two separate components are required to explain the $\mathrm{CO}$ lines observed with PACS. Emission from PDRs, produced along the envelope cavity walls due to UV-heating, was found to be the dominant component of the lower- $J$ lines $\left(J_{\text {up }}=14-20\right)$ accessible to PACS. The higher- $J$ CO lines $\left(J_{\text {up }} \gtrsim 25\right)$ were found to be dominated by the emission from the small-scale shocks along the cavity walls (van Kempen et al. 2010b). A more detailed modeling of the $\mathrm{CO}$ emission from three protostars with similar bolometric luminosities by Visser et al. (2012) essentially confirmed this picture, except that in these models the PDR component can dominate emission even for the high- $J$ CO lines, if the density of the envelope is sufficiently low. Thus, those authors tentatively suggested an evolutionary trend, in which the $\mathrm{CO}$ emission is dominated by shocks in the youngest source in their sample and by UV-heated gas in the oldest source with low envelope density (Visser et al. 2012). Herschel/PACS spectra of only a few sources have been studied so far, however. The analysis of a larger sample of protostars with a range in luminosity, evolutionary status, and envelope properties is required before the above suggestions can be confirmed. In this paper, we carry out such a study.

Here, we present FIR $(57-196 \mu \mathrm{m})$ spectra of 21 protostars in the Orion molecular clouds observed with Herschel/PACS. We obtained them as part of the Herschel Orion Protostar Survey (HOPS), an open time Key program with Herschel (Fischer et al. 2010; Stanke et al. 2010; Megeath et al. 2012). Our sample of protostars in Orion is the largest such sample at a common distance for which FIR spectra over the entire PACS wavelength range have been obtained and analyzed so far. Our analysis begins with a search for possible correlations between the observed $\mathrm{CO}$ emission properties and protostellar luminosity and evolutionary status. We then model the observed $\mathrm{CO}$ fluxes and line ratios to derive the excitation conditions of the warm and hot circumstellar gas and investigate the physical origin of $\mathrm{CO}$ emission from protostars. Our sample is described in Section 2. Observations and data reduction are described in Section 3. In Section 4, we present the observed properties of the $\mathrm{CO}$ emission from protostars in Orion and discuss various correlations found between CO emission and protostellar properties. In Section 5, we model the observed $\mathrm{CO}$ rotational diagrams to constrain the physical conditions of the emitting gas and discuss the results. The physical origin of the FIR CO emission from protostars is explored in Section 6. Finally, our conclusions are summarized in Section 7.

\section{HOPS SPECTROSCOPIC SAMPLE}

The HOPS sample for the PACS spectroscopic observations was selected from 300 Spitzer identified protostars in Orion with detectable $24 \mu \mathrm{m}$ emission (Kryukova et al. 2012; Megeath et al. 2012). We first selected objects with $F_{v} \geqslant 7 \mathrm{mJy}$ at $24 \mu \mathrm{m}$ with Class 0 or Class I spectral indices and other strong indications, such as deep silicate and ice features in the Spitzer Infrared Spectrograph (IRS) spectra, that they are indeed protostars. 
In this list we searched for objects that resemble, at infrared wavelengths, the nearly face-on protostar NGC 1333 IRAS 4B: evidence of compact $\left(\lesssim 10^{\prime \prime}\right.$ from the continuum position at $\gtrsim 24 \mu \mathrm{m}$ ) scattered light or $\mathrm{H}_{2}$ line emission at 2.3, 3.6, 4.5, or $24 \mu \mathrm{m}$ and no evidence for long jets or dust lanes suggestive of a near-edge-on orientation. Our motive here was for the sample to contain many objects for which our view to the dense inner regions would be relatively unextinguished. We initially identified 44 such protostars out of the parent sample of 300, all of which we observed with Spitzer IRS in its highest spectral resolution (D. M. Watson et al., in preparation). From these 44 sources, we chose 31 objects with bolometric luminosity uniformly sampled from 0.5 to $30 L_{\odot}$ to observe with PACS. An additional five objects with bolometric luminosity in the range of $\sim 20-200 L_{\odot}$ were added to the PACS target list without regard to orientation. In total, 36 protostars were observed with PACS in the spectroscopy mode as part of the HOPS program. Here we present PACS spectra of the brightest 21 sources in our sample, roughly uniformly sampling bolometric luminosity in the range of $\sim 1-200 L_{\odot}$. The FIR spectra of the full HOPS sample will be presented in a later paper. Due to the difficulty in determining the precise orientation of the protostars, many of the sources which were suspected to be close to face-on may actually be observed at a more inclined orientation. A future paper will use modeling of the spectral energy distributions (SEDs) as well as Hubble Space Telescope $1.6 \mu \mathrm{m}$ images to better constrain the inclinations for the HOPS protostars.

The observed SEDs of the protostars in our sample are presented in the Appendix. Through several ancillary observing programs that complement HOPS, we obtained extensive wavelength coverage for the sources in our sample resulting in well-sampled SEDs. From these SEDs, we computed the bolometric luminosity $\left(L_{\mathrm{bol}}\right)$ and bolometric temperature $\left(T_{\mathrm{bol}}\right)$ of each source. $L_{\text {bol }}$ was calculated by integrating the SED over frequency. Since the SEDs are sampled at a finite number of frequencies, we interpolated over the intervening frequencies following the midpoint interpolation method described in Enoch et al. (2009). The flux upper limits were removed and we interpolated over them before integration. Further, the SEDs were extrapolated from the non-upper limit flux at the longest observed wavelength (in most cases $870 \mu \mathrm{m}$ or $350 \mu \mathrm{m}$ ) using $F_{v} \propto v^{2} . T_{\text {bol }}$, which is defined as the temperature of a blackbody with the same mean frequency as the source SED, was computed from the mean frequency of the source SED, following the method of Myers \& Ladd (1993) and Enoch et al. (2009). Given our wide wavelength coverage, and, in particular, given that the peak of the SED is well characterized, our integration method accounts for most of the luminosity, and the $L_{\text {bol }}$ and $T_{\text {bol }}$ are well constrained. The dominant source of uncertainty in $L_{\text {bol }}$ and $T_{\text {bol }}$ is from the finite sampling errors, typically $\sim 10 \%-15 \%$. One of the sources in our sample, HOPS 369, has been modeled as a double source, i.e., a disk-dominated source plus a colder, envelope-dominated source by Adams et al. (2012; the source SOF 4 in the paper). The envelope source dominates the observed SED only at wavelengths $\gtrsim 37 \mu \mathrm{m}$ (see Figure 2 in Adams et al. 2012); thus we integrated the SED longward of $37 \mu \mathrm{m}$ to obtain $L_{\mathrm{bol}}$ and $T_{\mathrm{bol}}$ for HOPS 369 . The $L_{\mathrm{bol}}$ and $T_{\mathrm{bol}}$ computed for the protostars in our sample are listed in Table 1.

The distribution of the bolometric luminosities and temperatures of the protostars in our sample are presented in Figure 1. Protostars in our sample span a large range in $L_{\text {bol }}$; from $\sim 1.9 L_{\odot}$ to $217 L_{\odot}$. They generally are in different evolutionary phases. Protostars are generally classified into Class 0
Table 1

Protostellar Properties

\begin{tabular}{|c|c|c|c|c|c|}
\hline HOPS ID & R.A. (J2000) & Decl. (J2000) & $\begin{array}{l}L_{\text {bol }} \\
\left(L_{\odot}\right)\end{array}$ & $\begin{array}{l}T_{\text {bol }} \\
(\mathrm{K})\end{array}$ & $\begin{array}{c}\rho_{1}{ }^{\mathrm{a}} \\
\left(\times 10^{-14} \mathrm{~g} \mathrm{~cm}^{-3}\right)\end{array}$ \\
\hline 10 & 5359.005 & -55827.5 & 3 & 50 & 6 \\
\hline 11 & 53513.409 & -55758.1 & 8 & 59 & 37 \\
\hline 30 & 53444.062 & -54125.8 & 4 & 87 & 6 \\
\hline 32 & 53435.450 & -53959.1 & 2 & 60 & 6 \\
\hline 56 & 53519.466 & -51532.7 & 18 & 47 & 185 \\
\hline 60 & 53523.328 & -5123.06 & 21 & 60 & 6 \\
\hline 68 & 53524.305 & -5830.59 & 5 & 99 & 19 \\
\hline 84 & 53526.570 & -5355.12 & 45 & 93 & 6 \\
\hline 85 & 53528.183 & -5340.93 & 13 & 157 & 4 \\
\hline 87 & 53523.472 & -5128.70 & 27 & 39 & 185 \\
\hline 91 & 53518.914 & -5050.87 & 5 & 38 & 370 \\
\hline 108 & 53527.074 & -5100.37 & 57 & 41 & 185 \\
\hline 182 & 53618.833 & -62210.2 & 62 & 63 & 19 \\
\hline 203 & 53622.838 & -6466.20 & 15 & 45 & 74 \\
\hline 288 & 53955.944 & -73027.9 & 103 & 57 & 37 \\
\hline 310 & 54227.677 & -1201.00 & 11 & 55 & 19 \\
\hline 329 & 5471.606 & 01758.88 & 3 & 77 & 4 \\
\hline 343 & 54759.030 & 03532.86 & 4 & 88 & 6 \\
\hline 368 & 53524.725 & -51030.2 & 55 & 154 & 4 \\
\hline 369 & 53526.971 & -51017.1 & 20 & 42 & 90 \\
\hline 370 & 53527.629 & -5933.48 & 217 & 79 & 7 \\
\hline
\end{tabular}

Note. ${ }^{\text {a }}$ Fiducial envelope density, calculated at $1 \mathrm{AU}$ in the limit of no rotation (see Kenyon et al. 1993).

and Class I sources, two different, but related, evolutionary phases. Class 0 sources are the youngest protostars and have most of their mass still in the infalling envelope (André et al. 1993; André \& Montmerle 1994); they are highly obscured objects, invisible — in some cases — even at mid-infrared wavelengths. The more-evolved Class I sources have envelopes of mass less than the protostar itself and are visible in the nearinfrared wavelengths (Lada 1987; Wilking 1989). $T_{\text {bol }}$ of $70 \mathrm{~K}$ is generally taken as the dividing line between Class 0 and Class I sources (Chen et al. 1995; Evans et al. 2009). Based on this criterion, our sample has 13 Class 0 sources and 8 Class I sources.

To follow the evolution of the physical conditions in protostellar envelopes, we estimated the envelope densities of the protostars in our sample by modeling their SEDs. We assume that the density follows the rotating collapse solution of Terebey et al. (1984). While the density varies throughout the envelope, we adopt the approach of Kenyon et al. (1993) and use a fiducial density $\rho_{1}$, calculated at $1 \mathrm{AU}$ in the limit of no rotation, to track the envelope density. We computed $\rho_{1}$ by fitting the observed SEDs of the protostars with a large grid of models created with the Monte Carlo radiative transfer code of Whitney et al. (2003). The detailed description of the grid and the fitting procedure can be found in Tobin et al. (2008), Ali et al. (2010), and Fischer et al. (2010, 2012). For six sources in our sample (HOPS 60, 68, 108, 368, 369, 370), SED modeling results have already been published (Adams et al. 2012; Poteet et al. 2011); for these objects, the values of $\rho_{1}$ obtained from our modeling agreed with those published within a factor of $2-3$. For the composite SED of HOPS 369, we adopted the $\rho_{1}$ value derived for the envelope source by Adams et al. (2012). The envelope $\rho_{1}$ values for our sample are also listed in Table 1 and are plotted as a function of $L_{\mathrm{bol}}$ and $T_{\mathrm{bol}}$ in Figure 2. As can be readily seen, $\rho_{1}$ appears to be uncorrelated with $L_{\text {bol }}$. The Spearman rank coefficient, $r_{s}$, for the correlation is 0.27 and the probability that that this correlation could be produced by two random 

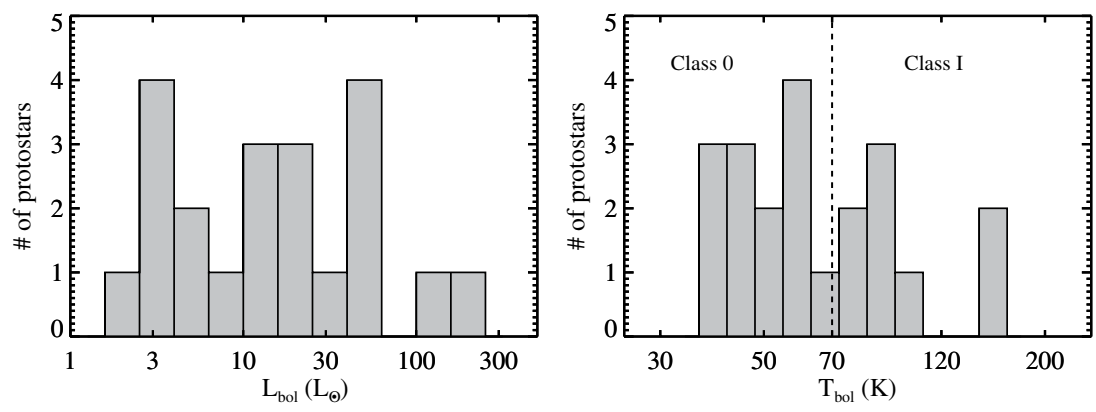

Figure 1. Distribution of $L_{\mathrm{bol}}$ and $T_{\mathrm{bol}}$ of the protostars for which PACS spectra are presented. $T_{\mathrm{bol}}$ of $70 \mathrm{~K}$ is generally taken as the dividing line between Class 0 and Class I sources (Chen et al. 1995; Evans et al. 2009).
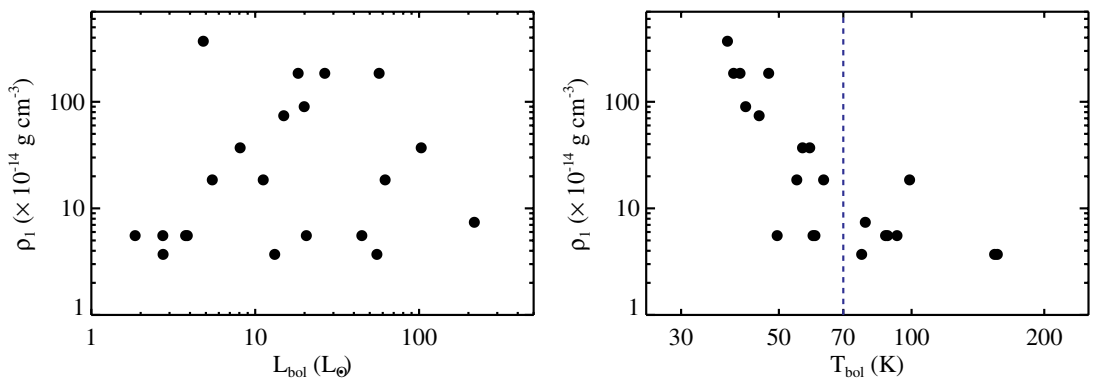

Figure 2. $\rho_{1}$ (see the text, Section 2) as a function of $L_{\mathrm{bol}}$ and $T_{\mathrm{bol}}$.

(A color version of this figure is available in the online journal.)

variables of the same sample size, $P\left(r_{s}\right)$, is $23 \%$. On the other hand, $\rho_{1}$ is tightly correlated with $T_{\text {bol }}$; the Spearman correlation coefficient, $r_{s}=-0.82$ and $P\left(r_{s}\right) \ll 0.001 \%$, indicating that the correlation is statistically significant. The envelope density generally decreases with increasing $T_{\mathrm{bol}}$, suggesting that $T_{\mathrm{bol}}$ is a good indicator of the evolutionary stage of the protostars in our sample (also see Chen et al. 1995; Evans et al. 2009).

\section{OBSERVATIONS AND DATA REDUCTION}

The log of Herschel PACS observations is provided in Table 2. All spectra were obtained in the range scan mode to achieve a total wavelength coverage of $57-196 \mu \mathrm{m}$. The wavelength range of $57-71 \mu \mathrm{m}$ was observed in the third grating order (B3A), the range $71-98 \mu \mathrm{m}$ in the second order (B2B), and the range $102-196 \mu \mathrm{m}$ in the first order (R1). Nyquist sampling, with grating step size of 6.25 spectral pixels was used to cover the broad wavelength ranges in each spectral band. Seventeen of the sources were observed in the pointed mode and four were observed in the full PACS spatial resolution mapping mode. The pointed observations of the bright sources $\left(F_{70} \gtrsim 10 \mathrm{Jy}\right)$ were carried out in the unchopped mode and those of the fainter sources $\left(F_{70} \lesssim 10 \mathrm{Jy}\right)$ in the chop/nod mode. The mapping observations were done in the unchopped mode. For the chop/nod observations, a maximum chopper throw of $6^{\prime}$ was used. For unchopped observations, offpositions free of cloud emission within $2^{\circ}$ from the target were used to subtract the instrument background. These emissionfree off-positions were determined from the CO $J=3-2$ maps of the region (J. Di Francesco et al., in preparation).

All spectra were reduced using the Herschel Interactive Processing Environment version 8.0 (Ott 2010). We used the pipeline scripts to obtain the final spectral maps over an area of $47^{\prime \prime} \times 47^{\prime \prime}$ centered on each source. An example of the PACS spectral map is shown in Figure 3, where the $5 \times 5$ spatial pixel ("spaxel") array and the spectrum corresponding to each spaxel is displayed. The telescope pointing in all our observations was good: we mapped the continuum in each of the PACS spectral bands and found that the continuum peak was well centered on the central spaxel within the pointing uncertainty of $\sim 2^{\prime \prime}$. The one-dimensional spectrum used in the analysis was extracted from the central spaxel $\left(99^{\prime \prime} 4 \times 99^{\prime \prime} 4\right)$ where the continuum emission from the protostar peaked. For the mapping observations, we extracted the one-dimensional spectrum within an 9 9.4 $^{\prime} \times 9$ 9.'4 aperture centered on the continuum peak. The FWHM of the spectrometer beam is a function of wavelength and ranges from $\sim 10^{\prime \prime}$ at $75 \mu \mathrm{m}$ to $\sim 12^{\prime \prime}$ at $160 \mu \mathrm{m}$ (see PACS Observer's Manual). We applied a wavelength-dependent PSFloss correction to the extracted spectrum to account for the different fractions of the PSF structure seen by the central spaxel at different wavelengths.

Representative FIR spectra $(57-196 \mu \mathrm{m})$ of a few sources in our sample are shown in Figure 4. At the distance of Orion $(\sim 420 \mathrm{pc})$, the extracted one-dimensional spectra correspond to the emission from within a radius of $\sim 2000$ AU from the protostars. Our PACS spectroscopic observations were designed to cover the rotational transitions in the ground vibrational state of CO ranging from $J=14-13$ to $J=46-45$. Several of these lines are seen in emission in the final extracted spectrum of most objects in our sample, indicating the presence of warm $\mathrm{CO}$ gas associated with these protostars. In four objects, HOPS 11, 30, 91, and 329, we do not detect any CO lines above the $3 \sigma$ rms level in the PACS wavelength range. In the remaining 17 objects, several $\mathrm{CO}$ lines are detected, ranging from 3 lines in HOPS 84 up to 30 lines in HOPS 370 and HOPS 108 (see Figure 4). We measured the fluxes of the observed CO lines in the spectra of the protostars by fitting them with Gaussian profiles. The measured CO line widths and the line sensitivities achieved from our observations are shown in Figure 5. 
Table 2

Log of PACS Spectroscopy Observations

\begin{tabular}{|c|c|c|c|c|c|c|}
\hline HOPS ID & ObsID & OD & Date & $\begin{array}{c}\text { Total Time } \\
\text { (s) }\end{array}$ & Observing Mode & $\begin{array}{c}\text { Primary Wavelength } \\
\text { Ranges Observed } \\
(\mu \mathrm{m})\end{array}$ \\
\hline \multirow[t]{2}{*}{10} & 1342215693 & 665 & 2011 Mar 10 & 3079 & Pointed/Unchopped & $57-71 \&$ 102-142 \\
\hline & 1342215694 & 665 & 2011 Mar 10 & 1930 & Pointed/Unchopped & $71-98$ \\
\hline \multirow[t]{2}{*}{11} & 1342204115 & 482 & 2010 Sep 7 & 5156 & Pointed/Chop-Nod & $71-98 \&$ 102-142 \\
\hline & 1342204116 & 482 & 2010 Sep 7 & 3369 & Pointed/Chop-Nod & $57-71$ \\
\hline \multirow[t]{2}{*}{30} & 1342215697 & 665 & 2011 Mar 10 & 3079 & Pointed/Unchopped & $57-71 \& 102-142$ \\
\hline & 1342215698 & 665 & 2011 Mar 10 & 1930 & Pointed/Unchopped & $71-98$ \\
\hline \multirow[t]{2}{*}{32} & 1342192115 & 303 & 2010 Mar 12 & 3129 & Pointed/Chop-Nod & $57-71$ \\
\hline & 1342192116 & 303 & 2010 Mar 12 & 5020 & Pointed/Chop-Nod & $71-98 \&$ 102-142 \\
\hline \multirow[t]{2}{*}{56} & 1342227334 & 833 & 2011 Aug 24 & 3079 & Pointed/Unchopped & $57-71 \& 102-142$ \\
\hline & 1342227335 & 833 & 2011 Aug 24 & 1930 & Pointed/Unchopped & $71-98$ \\
\hline \multirow[t]{4}{*}{60} & 1342227611 & 839 & 2011 Aug 30 & 8933 & Mapping/Unchopped & $57-71$ \\
\hline & 1342227613 & 839 & 2011 Aug 30 & 8933 & Mapping/Unchopped & $57-71$ \\
\hline & 1342227616 & 839 & 2011 Aug 30 & 6083 & Mapping/Unchopped & $71-98 \& 102-142$ \\
\hline & 1342227618 & 839 & 2011 Aug 30 & 6083 & Mapping/Unchopped & $71-98 \& 102-142$ \\
\hline \multirow[t]{2}{*}{68} & 1342226202 & 823 & 2011 Aug 14 & 3079 & Pointed/Unchopped & $57-71 \&$ 102-142 \\
\hline & 1342226203 & 823 & 2011 Aug 14 & 1930 & Pointed/Unchopped & $71-98$ \\
\hline \multirow[t]{2}{*}{84} & 1342227338 & 833 & 2011 Aug 25 & 3079 & Pointed/Unchopped & $57-71 \& 102-142$ \\
\hline & 1342227339 & 833 & 2011 Aug 25 & 1930 & Pointed/Unchopped & $71-98$ \\
\hline \multirow[t]{4}{*}{85} & 1342215657 & 664 & 2011 Mar 8 & 8933 & Mapping/Unchopped & $57-71$ \\
\hline & 1342215659 & 664 & 2011 Mar 8 & 8933 & Mapping/Unchopped & $57-71$ \\
\hline & 1342215662 & 664 & 2011 Mar 8 & 6083 & Mapping/Unchopped & $71-98 \& 102-142$ \\
\hline & 1342215664 & 664 & 2011 Mar 9 & 6083 & Mapping/Unchopped & $71-98 \&$ 102-142 \\
\hline \multirow[t]{2}{*}{87} & 1342215650 & 664 & 2011 Mar 8 & 3079 & Pointed/Unchopped & $57-71 \& 102-142$ \\
\hline & 1342215651 & 664 & 2011 Mar 8 & 1930 & Pointed/Unchopped & $71-98$ \\
\hline \multirow[t]{2}{*}{91} & 1342215654 & 664 & 2011 Mar 8 & 3079 & Pointed/Unchopped & $57-71 \& 102-142$ \\
\hline & 1342215655 & 664 & 2011 Mar 8 & 1930 & Pointed/Unchopped & $71-98$ \\
\hline \multirow[t]{2}{*}{108} & 1342239690 & 1020 & 2012 Feb 27 & 3079 & Pointed/Unchopped & $57-71 \& 102-142$ \\
\hline & 1342239693 & 1020 & 2012 Feb 27 & 1930 & Pointed/Unchopped & 71-98 \\
\hline \multirow[t]{2}{*}{182} & 1342226753 & 826 & 2011 Aug 18 & 3079 & Pointed/Unchopped & $57-71 \& 102-142$ \\
\hline & 1342226754 & 826 & 2011 Aug 18 & 1930 & Pointed/Unchopped & $71-98$ \\
\hline \multirow[t]{2}{*}{203} & 1342191363 & 290 & 2010 Feb 28 & 3129 & Pointed/Chop-Nod & $57-71$ \\
\hline & 1342191364 & 290 & 2010 Feb 28 & 5020 & Pointed/Chop-Nod & $71-98 \& 102-142$ \\
\hline \multirow[t]{4}{*}{288} & 1342227754 & 838 & 2011 Aug 29 & 8933 & Mapping/Unchopped & $57-71$ \\
\hline & 1342227756 & 838 & 2011 Aug 29 & 8933 & Mapping/Unchopped & $57-71$ \\
\hline & 1342227759 & 838 & 2011 Aug 29 & 6083 & Mapping/Unchopped & $71-98 \& 102-142$ \\
\hline & 1342227761 & 838 & 2011 Aug 29 & 6083 & Mapping/Unchopped & $71-98 \& 102-142$ \\
\hline \multirow[t]{2}{*}{310} & 1342215689 & 665 & 2011 Mar 10 & 3079 & Pointed/Unchopped & $57-71 \&$ 102-142 \\
\hline & 1342215689 & 665 & 2011 Mar 10 & 1930 & Pointed/Unchopped & $71-98$ \\
\hline \multirow[t]{2}{*}{329} & 1342217855 & 690 & 2011 Apr 4 & 3079 & Pointed/Unchopped & $57-71 \& 102-142$ \\
\hline & 1342217856 & 690 & 2011 Apr 4 & 1930 & Pointed/Unchopped & $71-98$ \\
\hline \multirow[t]{2}{*}{343} & 1342217851 & 690 & 2011 Apr 4 & 3079 & Pointed/Unchopped & $57-71 \& 102-142$ \\
\hline & 1342217852 & 690 & 2011 Apr 4 & 1930 & Pointed/Unchopped & $71-98$ \\
\hline \multirow[t]{2}{*}{368} & 1342227340 & 833 & 2011 Aug 25 & 3079 & Pointed/Unchopped & $57-71 \& 102-142$ \\
\hline & 1342227341 & 833 & 2011 Aug 25 & 1930 & Pointed/Unchopped & 71-98 \\
\hline \multirow[t]{2}{*}{369} & 1342227344 & 833 & 2011 Aug 25 & 3079 & Pointed/Unchopped & $57-71 \& 102-142$ \\
\hline & 1342227345 & 833 & 2011 Aug 25 & 1930 & Pointed/Unchopped & $71-98$ \\
\hline 370 & 1342227764 & 838 & 2011 Aug 29 & 8933 & Mapping/Unchopped & $57-71$ \\
\hline & 1342227766 & 838 & 2011 Aug 30 & 8933 & Mapping/Unchopped & $57-71$ \\
\hline & 1342227769 & 838 & 2011 Aug 30 & 6083 & Mapping/Unchopped & $71-98 \& 102-142$ \\
\hline & 1342227771 & 838 & 2011 Aug 30 & 6083 & Mapping/Unchopped & $71-98 \& 102-142$ \\
\hline
\end{tabular}

\section{OBSERVED PROPERTIES OF FIR CO EMISSION FROM PROTOSTARS}

\subsection{CO Excitation Diagrams and Rotational Temperatures}

Since several CO lines are detected for many objects in the PACS wavelength range, it is useful to construct a rotational excitation diagram, which shows the relative populations of $\mathrm{CO}$ rotational states derived from the observed line fluxes. In this diagram, the natural logarithm of the detected number of $\mathrm{CO}$ molecules in a rotational state divided by the degeneracy of that state, $\ln \left(N_{J} / g_{J}\right)$, is plotted as a function of the energy, $E_{J}$ (see, e.g., Goldsmith \& Langer 1999). If the CO emission is optically thin, then the number of $\mathrm{CO}$ molecules in the $J$ th rotational state is given by

$$
N_{J}=\left(4 \pi d^{2} F_{J, J-1}\right) /\left(h v_{J, J-1} A_{J, J-1}\right),
$$

where $F_{J, J-1}, v_{J, J-1}$ and $A_{J, J-1}$ are the line flux, frequency, and Einstein A-coefficient, respectively, corresponding to the transition $J \rightarrow J-1$ and $d$ is the distance to the source. The FIR CO lines observed with PACS are likely to be optically 


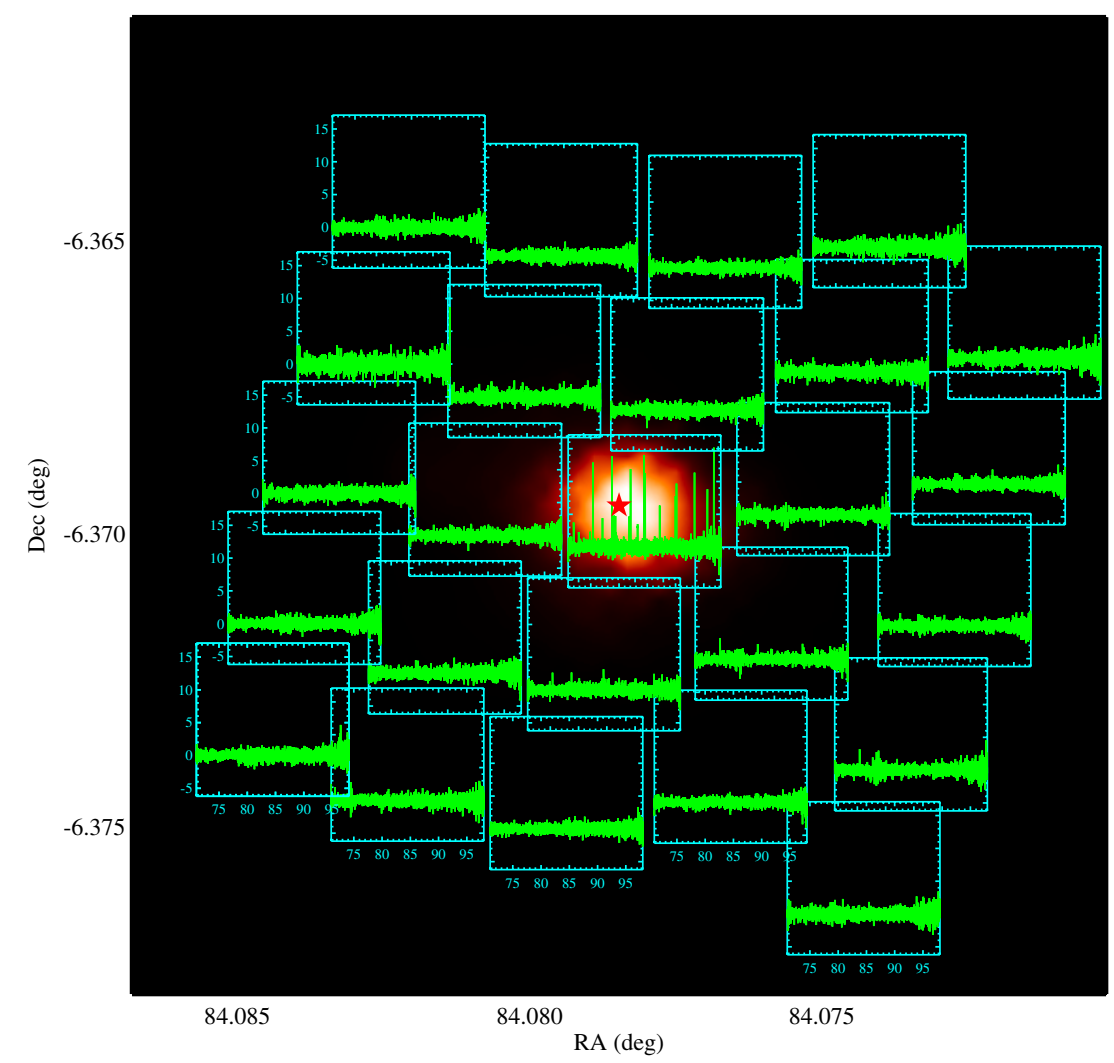

Figure 3. PACS spectral map of HOPS 182 in the B2B $(72-98 \mu \mathrm{m})$ spectral band. Within the square boxes, which represent the $5 \times 5$ spaxel array, the observed spectra at that location are shown. The PACS spectroscopic field is overlaid on the PACS $70 \mu \mathrm{m}$ image of HOPS 182 obtained as part of the HOPS program. The star symbol represents the MIPS $24 \mu \mathrm{m}$ coordinates of HOPS 182, which was used for telescope pointing.

(A color version of this figure is available in the online journal.)

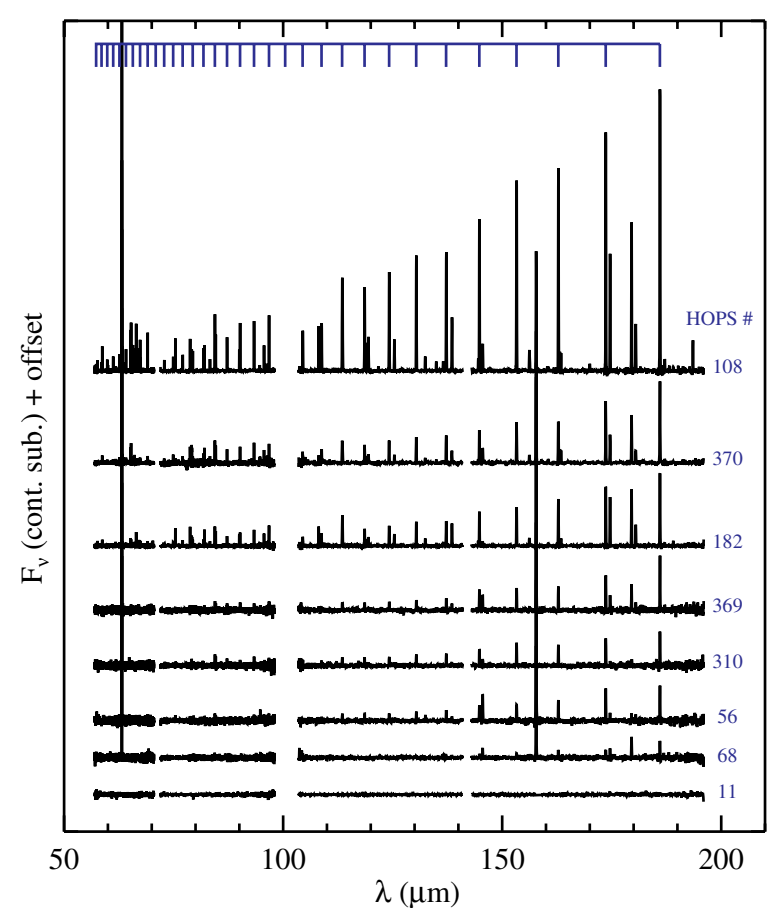

Figure 4. Continuum-subtracted PACS spectra of eight protostars which are representative of the entire sample. The central wavelengths of the observed $\mathrm{CO}$ lines $\left(J_{\text {up }}=14-46\right)$ are indicated at the top. The continuum has been subtracted by applying a median filter to the spectra. An arbitrary offset has been added to the spectra to separate them in the plot.

(A color version of this figure is available in the online journal.) thin if the size of the emitting region is sufficiently large $\left(\gtrsim 1^{\prime \prime}\right)$. Figure 6 shows the optical depths of the CO $J=14-13$ line observed toward the protostars in our sample for three assumed angular sizes of emission. To compute the optical depth, we assumed an excitation temperature of $300 \mathrm{~K}$ and a line width (FWHM) of $10 \mathrm{~km} \mathrm{~s}^{-1}$. The observed CO $J=14-13$ transition, which is the lowest excitation line observed and the most likely one to be optically thick, has $\tau \gg 1$ only if the size of the emitting region is $\ll 1^{\prime \prime}(\ll 420 \mathrm{AU}$ at the distance of Orion). The extent of the observed $\mathrm{CO}$ emission is likely to be much larger $(\sim 1000 \mathrm{AU})$ than this (Visser et al. 2012). Moreover, Yildiz et al. (2012) have shown that even for the CO $J=6-5$ line the optical depths are low $(\tau<2)$ for broad velocity component $\left(\right.$ FWHM $\left.\gtrsim 10-15 \mathrm{~km} \mathrm{~s}^{-1}\right)$. The higher excitation $\left(J_{\text {up }} \geqslant 14\right)$ CO lines observed with PACS are likely to be broader than this, which would make the line optical depths even lower. Thus, the optically thin assumption appears valid and the above equation can be used to compute $N_{J}$ and to construct the rotational diagrams. The $\mathrm{CO}$ rotational diagrams for the 17 protostars with $\mathrm{CO}$ detections are shown in Figure 7.

The observed rotational diagrams of the protostars typically show a positive curvature, which is more apparent in cases where more than 10 lines are detected. The rotational diagrams of a few other protostars whose Herschel/PACS spectra have been published (van Kempen et al. 2010b; Fich et al. 2010; Herczeg et al. 2012) also show similar positive curvature as noted by Neufeld (2012). This means that the rotational temperature defined as $T_{\text {rot }}=-\left(k d \ln \left[N_{J} / g_{J}\right] / d E_{J}\right)^{-1}$ increases monotonically with $E_{J}$. An average rotational temperature can 

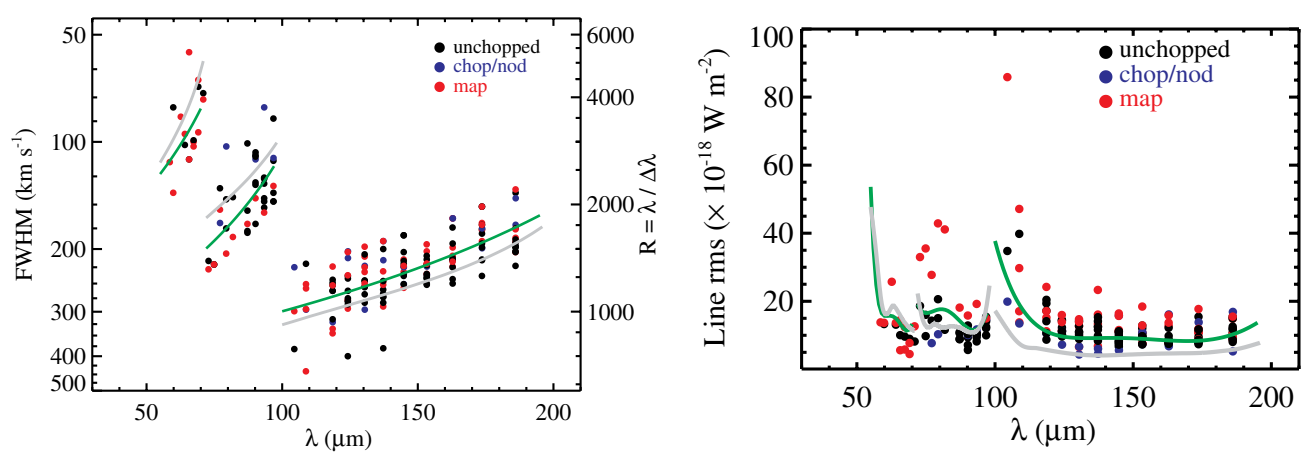

Figure 5. Measured CO line widths (left) and $1 \sigma$ line sensitivity of our observations (right). Lines detected at $3 \sigma$ level or higher are shown. Pointed/unchopped (black solid circles), pointed/chop-nod (blue circles), and mapping/unchopped (red circles) observations are identified. The gray solid lines correspond to HSpot predictions for our observational set up. The green solid lines are fits to the observed points in different spectral bands.

(A color version of this figure is available in the online journal.)

Table 3

CO Rotational Temperatures and Luminosity

\begin{tabular}{|c|c|c|c|c|c|c|}
\hline HOPS ID & $\begin{array}{l}T_{\mathrm{LR} 1} \\
(\mathrm{~K})\end{array}$ & $\begin{array}{l}T_{\mathrm{SR} 1} \\
(\mathrm{~K})\end{array}$ & $\begin{array}{l}T_{\mathrm{R} 1} \\
(\mathrm{~K})\end{array}$ & $\begin{array}{l}T_{\mathrm{B} 2 \mathrm{~B}} \\
(\mathrm{~K})\end{array}$ & $\begin{array}{l}T_{\mathrm{B} 3 \mathrm{~A}} \\
(\mathrm{~K})\end{array}$ & $\begin{array}{c}L_{\mathrm{CO}} \\
\left(\times 10^{-3} L_{\odot}\right)\end{array}$ \\
\hline 10 & $238 \pm 44$ & $391 \pm 280$ & $315 \pm 43$ & $\ldots$ & $\ldots$ & 1.3 \\
\hline 11 & $\ldots$ & $\ldots$ & $\ldots$ & $\ldots$ & $\ldots$ & $\leqslant 0.7$ \\
\hline 30 & $\ldots$ & $\ldots$ & $\ldots$ & $\ldots$ & $\ldots$ & $\leqslant 0.8$ \\
\hline 32 & $307 \pm 46$ & $282 \pm 91$ & $275 \pm 19$ & $\ldots$ & $\ldots$ & 1.8 \\
\hline 56 & $218 \pm 10$ & $338 \pm 58$ & $262 \pm 11$ & $550 \pm 361$ & $\ldots$ & 8.2 \\
\hline 60 & $221 \pm 11$ & $303 \pm 27$ & $294 \pm 13$ & $\ldots$ & $\ldots$ & 6.2 \\
\hline 68 & $144 \pm 11$ & $\ldots$ & $144 \pm 11$ & $\ldots$ & $\ldots$ & 1.6 \\
\hline 84 & $186 \pm 49$ & $\ldots$ & $186 \pm 49$ & $\ldots$ & $\ldots$ & 0.7 \\
\hline 85 & $186 \pm 37$ & $\ldots$ & $270 \pm 34$ & $\ldots$ & $\ldots$ & 1.2 \\
\hline 87 & $270 \pm 19$ & $307 \pm 49$ & $316 \pm 13$ & $575 \pm 475$ & $\ldots$ & 7.6 \\
\hline 91 & $\ldots$ & $\ldots$ & $\ldots$ & $\ldots$ & $\ldots$ & $\leqslant 0.8$ \\
\hline 108 & $290 \pm 3$ & $410 \pm 4$ & $344 \pm 12$ & $734 \pm 30$ & $1076 \pm 25$ & 238.0 \\
\hline 182 & $230 \pm 3$ & $413 \pm 15$ & $331 \pm 20$ & $703 \pm 27$ & $1220 \pm 223$ & 48.4 \\
\hline 203 & $322 \pm 34$ & $443 \pm 35$ & $350 \pm 16$ & $773 \pm 96$ & $\ldots$ & 9.7 \\
\hline 288 & $234 \pm 11$ & $362 \pm 45$ & $315 \pm 19$ & . & $\ldots$ & 10.0 \\
\hline 310 & $252 \pm 11$ & $375 \pm 49$ & $268 \pm 6$ & $924 \pm 152$ & $\ldots$ & 9.8 \\
\hline 329 & $\ldots$ & $\ldots$ & $\ldots$ & $\ldots$ & $\ldots$ & $\leqslant 0.7$ \\
\hline 343 & $260 \pm 51$ & $570 \pm 417$ & $375 \pm 43$ & $\ldots$ & $\ldots$ & 2.0 \\
\hline 368 & $193 \pm 8$ & $386 \pm 86$ & $262 \pm 17$ & $739 \pm 313$ & $\ldots$ & 6.4 \\
\hline 369 & $191 \pm 6$ & $398 \pm 52$ & $264 \pm 18$ & $807 \pm 168$ & $\ldots$ & 11.0 \\
\hline 370 & $239 \pm 4$ & $374 \pm 17$ & $332 \pm 13$ & $807 \pm 42$ & $1775 \pm 271$ & 58.0 \\
\hline
\end{tabular}

Notes. $T_{\mathrm{LR} 1}$ is computed for the CO lines in the range $J_{\mathrm{up}}=14-18, T_{\mathrm{SR} 1}$ for $J_{\text {up }}=19-25, T_{\mathrm{R} 1}$ for $J_{\text {up }}=14-25, T_{\mathrm{B} 2 \mathrm{~B}}$ for $J_{\text {up }}=27-36$, and $T_{\mathrm{B} 3 \mathrm{~A}}$ for $J_{\text {up }}=37-46$.

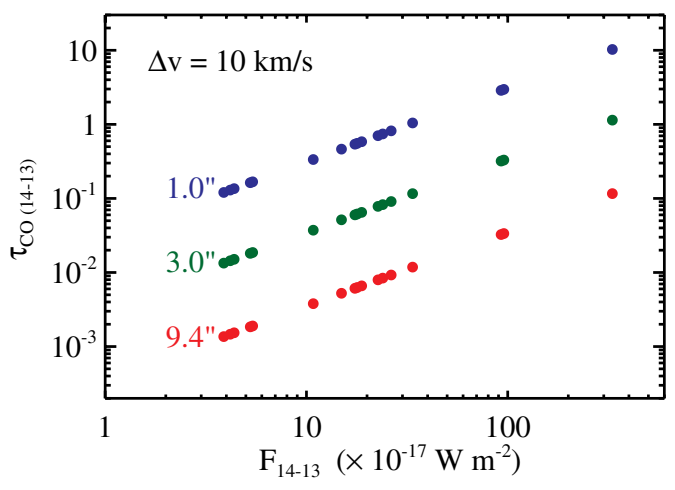

Figure 6. Optical depth, $\tau$, of the CO $J=14-13$ line as a function of observed line flux for three angular sizes (for $d=420 \mathrm{pc}$ to Orion) of the emitting region. The optical depths shown are for a CO line width (FWHM) of $10 \mathrm{~km} \mathrm{~s}^{-1}$.

(A color version of this figure is available in the online journal.) be computed for a relatively small range in $E_{J}$ by fitting a straight line to the points in the observed rotational diagram. We begin our analysis by computing the average slopes of the observed rotational diagrams for the PACS spectral bands R1 $\left(102-190 \mu \mathrm{m} ; 550 \mathrm{~K} \leqslant E_{J} / k \leqslant 1800 \mathrm{~K} ; J_{\text {up }}=14-25\right)$, B2B $\left(72-98 \mu \mathrm{m} ; 2000 \mathrm{~K} \leqslant E_{J} / k \leqslant 3700 \mathrm{~K} ; J_{\mathrm{up}}=27-36\right)$, and B3A $\left(57-71 \mu \mathrm{m} ; 3700 \mathrm{~K} \leqslant E_{J} / k \leqslant 6000 \mathrm{~K} ; J_{\text {up }}=37-46\right)$. The best linear fit for the average slope was obtained by minimizing $\chi^{2}$; from these slopes, we obtained the average rotational temperatures, $T_{\mathrm{R} 1}, T_{\mathrm{B} 2 \mathrm{~B}}$, and $T_{\mathrm{B} 3 \mathrm{~A}}$ which are shown in Figure 7 and are listed in Table 3. This method of computing $T_{\text {rot }}$ for $\mathrm{CO}$ lines observed within the same spectral band has the advantage of minimizing possible systematic uncertainties introduced by any mismatch in the relative flux calibration between different spectral bands.

Figure 8 shows the distribution of the rotational temperatures $T_{\mathrm{R} 1}, T_{\mathrm{B} 2 \mathrm{~B}}$, and $T_{\mathrm{B} 3 \mathrm{~A}} . T_{\mathrm{R} 1}$ has a narrow distribution ranging from 

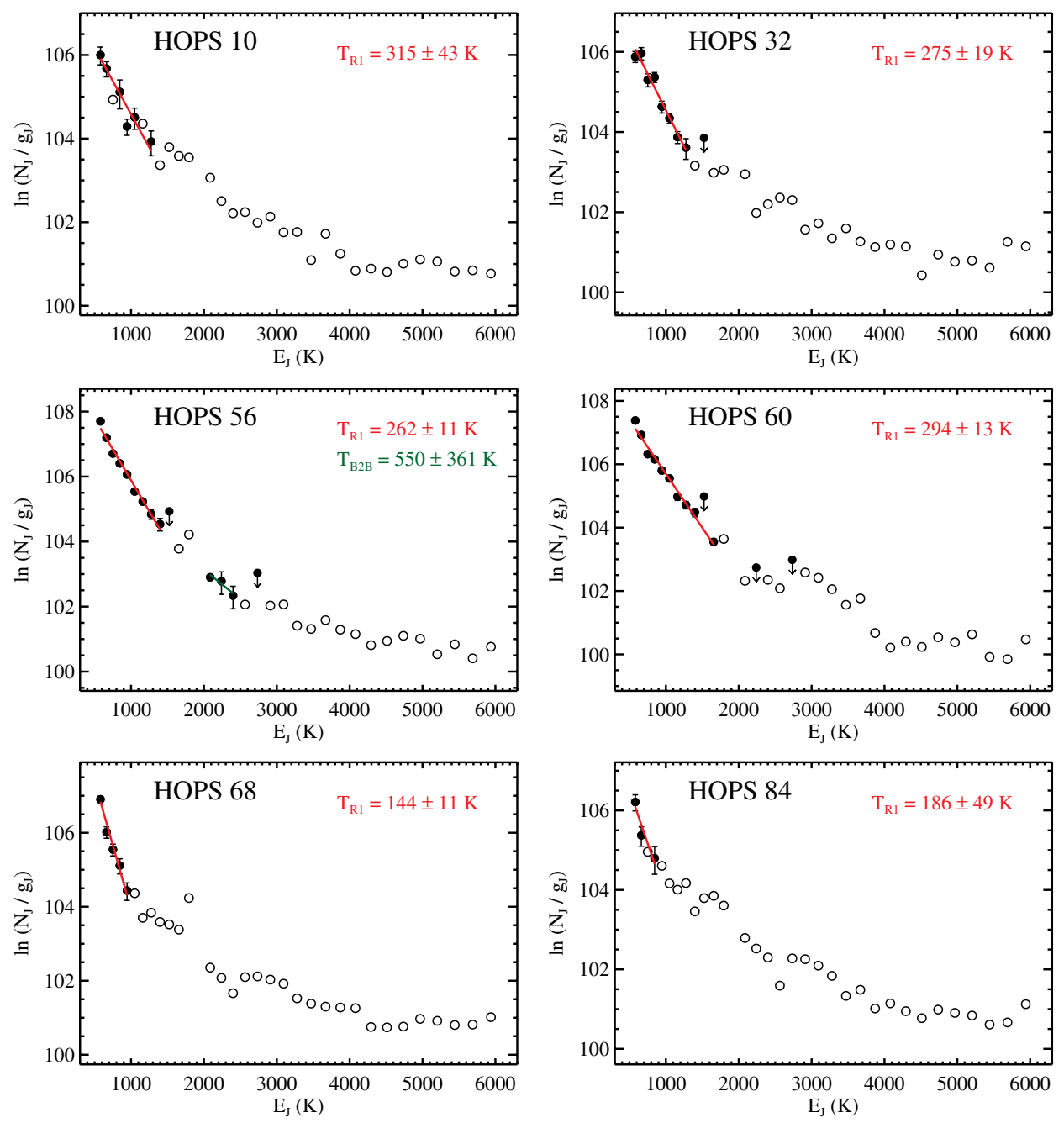

Figure 7. CO rotational excitation diagrams for the 17 protostars toward which $\mathrm{CO}$ lines are detected. Linear fits to the $\mathrm{CO}$ lines in the $\mathrm{R} 1\left(550 \mathrm{~K} \leqslant E_{J} / k \leqslant 1800 \mathrm{~K}\right.$; $\left.J_{\mathrm{up}}=14-25\right)$ band (red solid line), the B2B $\left(2000 \mathrm{~K} \leqslant E_{J} / k \leqslant 3700 \mathrm{~K} ; J_{\mathrm{up}}=27-36\right)$ band (green solid line), and the B3A $\left(3700 \mathrm{~K} \leqslant E_{J} / k \leqslant 6000 \mathrm{~K} ; J_{\mathrm{up}}=\right.$ $37-46$ ) band (blue solid line) and the average rotational temperatures derived, $T_{\mathrm{R} 1}, T_{\mathrm{B} 2 \mathrm{~B}}$, and $T_{\mathrm{B} 3 \mathrm{~A}}$ are shown. Downward arrows indicate upper limits to the fluxes of CO lines that are blended with a nearby line. Open circles correspond to $3 \sigma$ upper limits for the non-detections. Sources in which no CO lines are detected are not shown.

(A color version of this figure is available in the online journal.)

$144 \mathrm{~K}$ to $375 \mathrm{~K}$ with a median value of $294 \mathrm{~K}$. The uncertainties in $T_{\mathrm{R} 1}$ are relatively small with an average value of $21 \mathrm{~K} . T_{\mathrm{B} 2 \mathrm{~B}}$ ranges from $550 \mathrm{~K}$ to $924 \mathrm{~K}$ and has a median value of $739 \mathrm{~K}$; it is less well determined and has relatively large uncertainties (185 K). $T_{\mathrm{B} 3 \mathrm{~A}}$ could be determined only for three sources and ranges from $1076 \mathrm{~K}$ to $1775 \mathrm{~K}$ (see Table 3 and Figure 8). Since, as pointed out above, $T_{\text {rot }}$ increases monotonically with $E_{J}$ (or $J$ ), the estimated average rotational temperatures generally increase with the number of lines detected in a spectral band. The spread in rotational temperatures listed in Table 3 is partly due to this behavior. For instance, for 11 sources for which more than $9 \mathrm{CO}$ lines are detected in the $\mathrm{R} 1$ band, the corresponding $T_{\mathrm{R} 1}$ values show a much smaller range (262-350 K with a median value of $315 \mathrm{~K})$. Similarly, for six sources in which five or more lines are detected in the $\mathrm{B} 2 \mathrm{~B}$ band, the corresponding $T_{\mathrm{B} 2 \mathrm{~B}}$ values ranges from $703 \mathrm{~K}$ to $924 \mathrm{~K}$. Thus, the observed $\mathrm{CO}$ rotational temperatures, in particular $T_{\mathrm{R} 1}$, are very similar for the protostars in our sample.

We also computed the average rotational temperatures separately for the PACS spectral bands Long R1 (142-190 $\mu \mathrm{m}$; $\left.550 \mathrm{~K} \leqslant E_{J} / k \leqslant 1000 \mathrm{~K} ; J_{\text {up }}=14-18\right)$ and Short R1 $\left(103-140 \mu \mathrm{m} ; 1000 \mathrm{~K} \leqslant E_{J} / k \leqslant 2000 \mathrm{~K} ; J_{\text {up }}=19-25\right)$ which we will call $T_{\mathrm{LR} 1}$ and $T_{\mathrm{SR} 1}$. The rotational temperatures are computed only if there are more than two lines detected in a given spectral band. The distribution of $T_{\mathrm{LR} 1}$ and $T_{\mathrm{SR} 1}$ for sources where both these temperatures could be determined is presented in Figure 9. Although there is some overlap between the derived values of $T_{\mathrm{LR} 1}$ and $T_{\mathrm{SR} 1}$, for most objects there is a significant difference between the two. The median value of $T_{\mathrm{LR} 1}$ is $239 \mathrm{~K}$ and that for $T_{\mathrm{SR} 1}$ is $386 \mathrm{~K}$; this difference is more than what can be accounted for by the uncertainties in these quantities. This difference suggests that even in the R1 spectral band there is a significant curvature in the rotational diagram for most objects indicating that even the mid- $J$ CO lines $\left(J_{\text {up }}=14\right.$ to $\left.J_{\text {up }}=25\right)$ observed in the PACS R1 spectral band cannot be explained by a single-temperature component in local thermodynamic equilibrium (LTE).

\subsection{Correlation with Protostellar Properties}

The CO emission lines detected in the PACS wavelength range have their origin in the warm gas in the immediate vicinity (i.e., at projected distance $\lesssim 2000 \mathrm{AU}$ at $420 \mathrm{pc}$ ) of the protostars. 

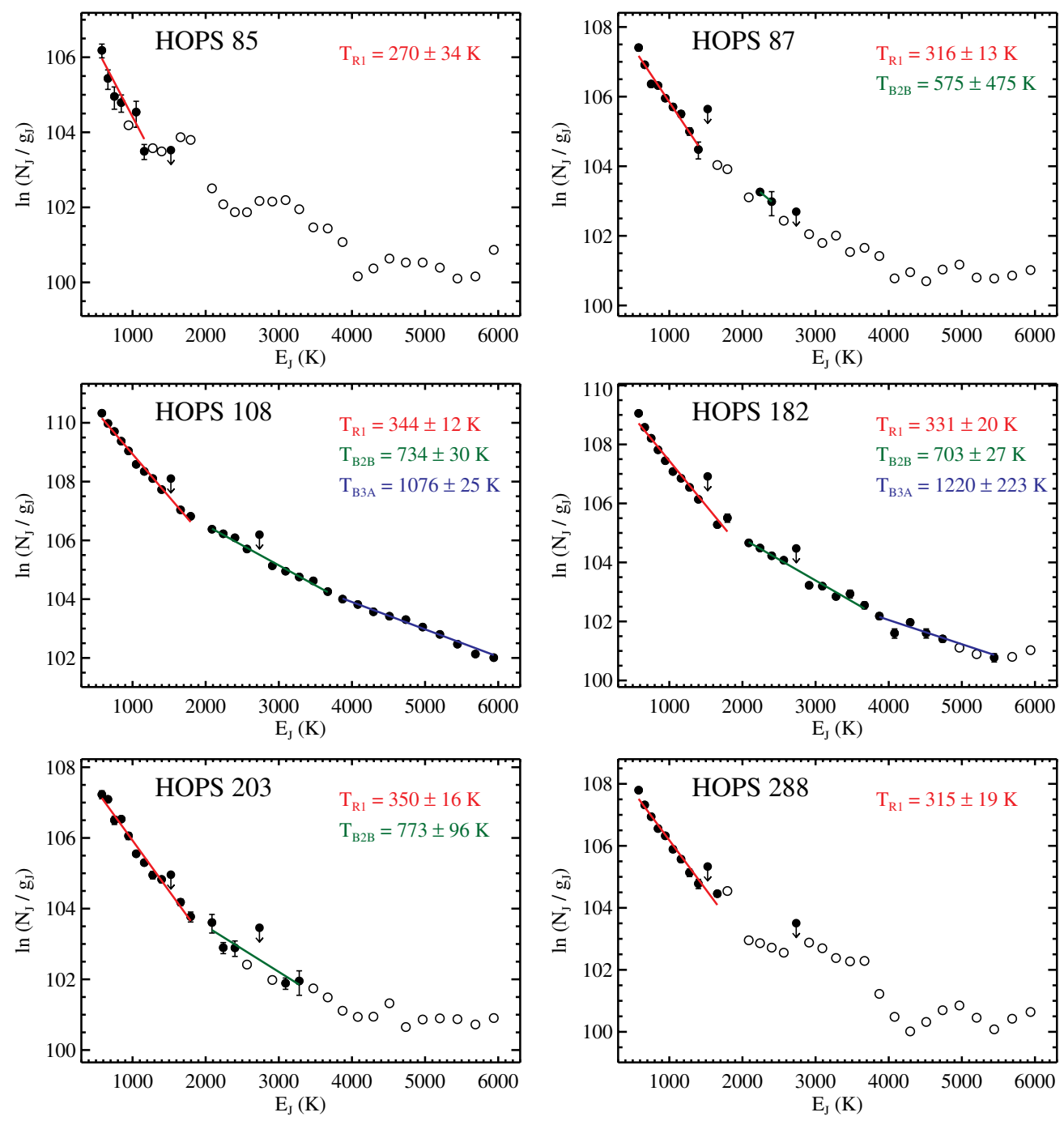

Figure 7. (Continued)

Since the energy that goes into the heating of the circumstellar gas must be derived ultimately from the central protostar, it is instructive to search for possible correlations between the observed characteristics of the $\mathrm{CO}$ emission and protostellar properties.

We first computed the total observed $\mathrm{CO}$ luminosity, $L_{\mathrm{CO}}$, by adding up the fluxes of all the lines detected with $\mathrm{S} / \mathrm{N} \geqslant 3 \sigma . L_{\mathrm{CO}}$ is plotted as a function of $L_{\mathrm{bol}}, T_{\mathrm{bol}}$, and the envelope density at $1 \mathrm{AU}, \rho_{1}$, for all the protostars in our sample in Figure 10. For sources where no CO lines were detected within the PACS wavelength range, an upper limit to $L_{\mathrm{CO}}$ was computed by adding up the $3 \sigma$ detection limit at the location of each CO line. To carry out a correlation analysis accommodating the upper limits, we used the Astronomical Survival Analysis (ASURV) package (Lavalley et al. 1992) that implements the methods presented in Feigelson \& Nelson (1985) and Isobe et al. (1986). We used the generalized Kendall's tau and Spearman's rank order tests to compute the correlation probabilities between $L_{\mathrm{CO}}$ and the protostellar properties. These tests show that $L_{\mathrm{CO}}$ is tightly correlated with $L_{\mathrm{bol}}$. The associated Spearman rank correlation coefficient, $r_{s}=0.71$, and the probability that $L_{\mathrm{CO}}$ and $L_{\text {bol }}$ are uncorrelated is $0.2 \%$; the generalized Kendall's tau test gives this probability to be $0.3 \%$. On the other hand, no statistically significant correlation is found between $L_{\mathrm{CO}}$ and the protostellar evolutionary indicators $T_{\text {bol }}$ (Spearman probability $=17 \%$ and Kendall's tau probability $=$ $18 \%$ ) or $\rho_{1}$ (Spearman probability $=8 \%$ and Kendall's tau probability $=9 \%)$.

CO rotational temperatures $T_{\mathrm{R} 1}$ and $T_{\mathrm{B} 2 \mathrm{~B}}$ (see Section 4.1) are plotted as a function of $L_{\mathrm{bol}}, T_{\mathrm{bol}}$, and $\rho_{1}$ for the protostars in our sample in Figure 11. $T_{\mathrm{R} 1}$ is uncorrelated with $L_{\text {bol }}$ and remains constant over more than two orders of magnitude in $L_{\mathrm{bol}} . T_{\mathrm{B} 2 \mathrm{~B}}$ could be determined only for protostars with $L_{\text {bol }} \gtrsim 10 L_{\odot}$ as high- $J$ CO lines $\left(E_{J} \geqslant 2000 K\right)$ are detected only for these objects. $T_{\mathrm{B} 2 \mathrm{~B}}$ also appears to be uncorrelated with $L_{\mathrm{bol}}$ and within the uncertainties it remains roughly constant over a smaller range in $L_{\text {bol }}$. Further, $T_{\mathrm{R} 1}$ and $T_{\mathrm{B} 2 \mathrm{~B}}$ are found to be uncorrelated with the $T_{\text {bol }}$ and $\rho_{1}$ values of the protostars, as shown in Figure 11. Additionally, Figure 12 shows that $T_{\mathrm{LR} 1}$ and $T_{\mathrm{SR} 1}$ also are uncorrelated with protostellar luminosity; they remain more or less constant over a large range in $L_{\mathrm{bol}}$. This behavior demonstrates that irrespective of the range in $E_{J}$ (or $J$ ) over which the average rotational temperatures are computed, they remain independent of $L_{\text {bol }}$.

In summary, the observed CO line fluxes and luminosities scale with $L_{\text {bol }}$. The relative line fluxes (as quantified by the rotational temperatures), however, appear independent of $L_{\text {bol }}$. Both the CO line luminosities and the CO rotational 

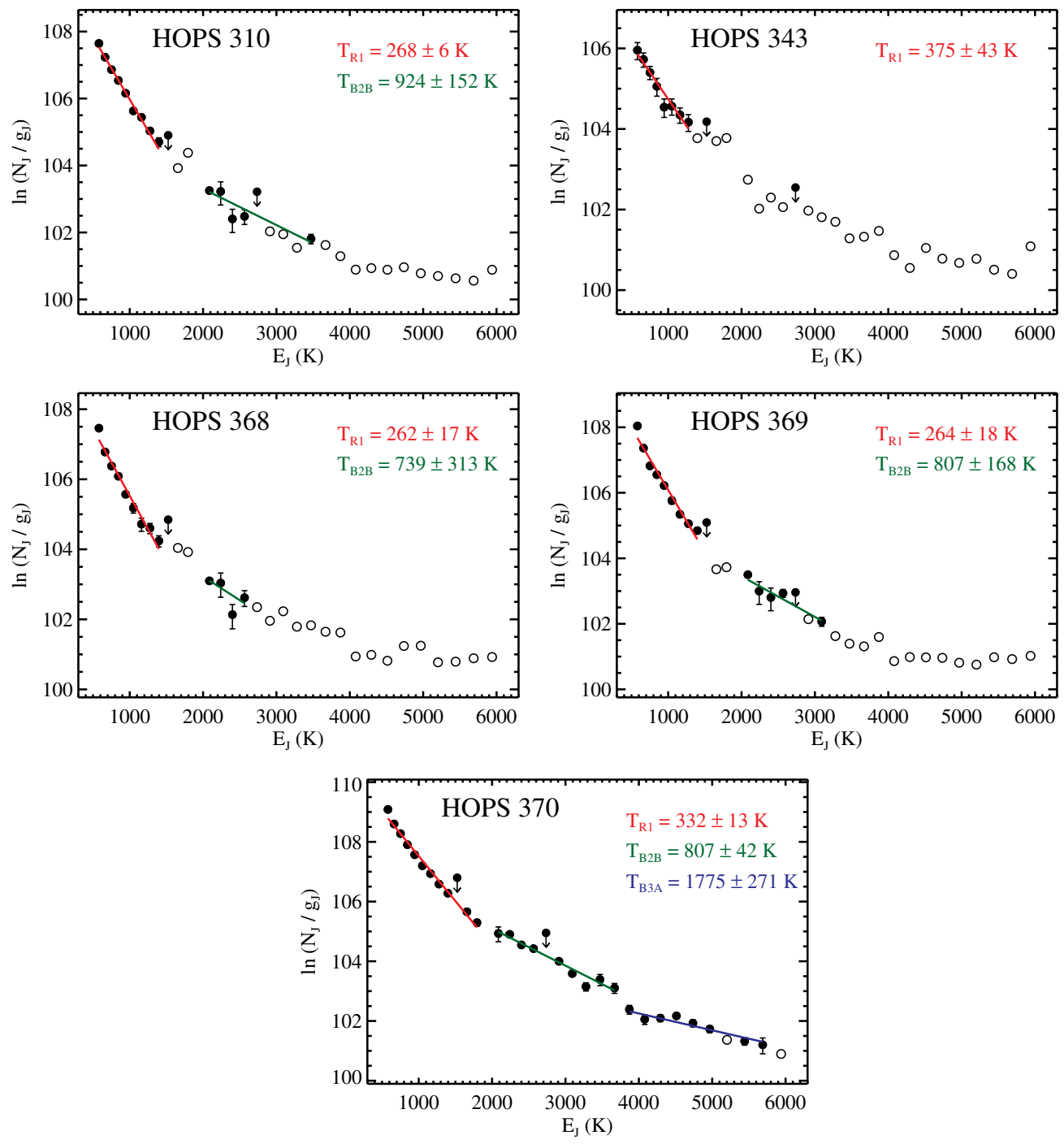

Figure 7. (Continued)

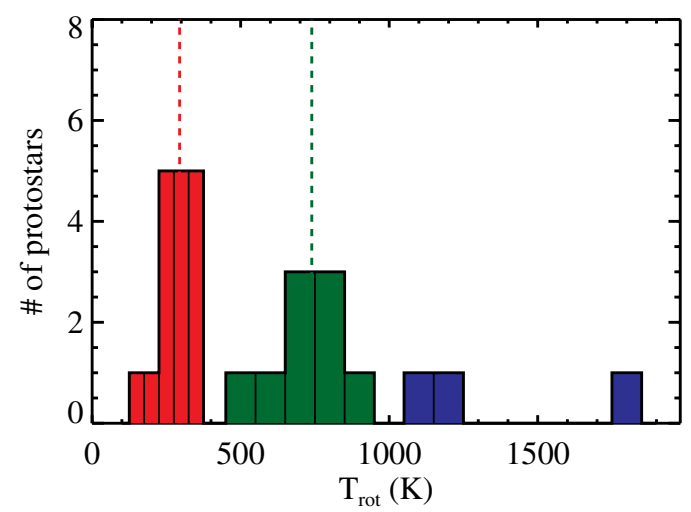

Figure 8. Distribution of the average $\mathrm{CO}$ rotational temperatures, $T_{\mathrm{R} 1}$ (red), $T_{\mathrm{B} 2 \mathrm{~B}}$ (green), and $T_{\mathrm{B} 3 \mathrm{~A}}$ (blue). Dashed lines indicate median values of $T_{\mathrm{R} 1}$ (red) and $T_{\mathrm{B} 2 \mathrm{~B}}$ (green).

(A color version of this figure is available in the online journal.)

temperatures show no significant correlation with evolutionary indicators or envelope properties such as $T_{\text {bol }}$ and $\rho_{1}$.

\subsection{CO Emission from an Outflow Lobe}

In the PACS field observed toward one of the sources in our sample, HOPS 87, we detected CO emission from the extended

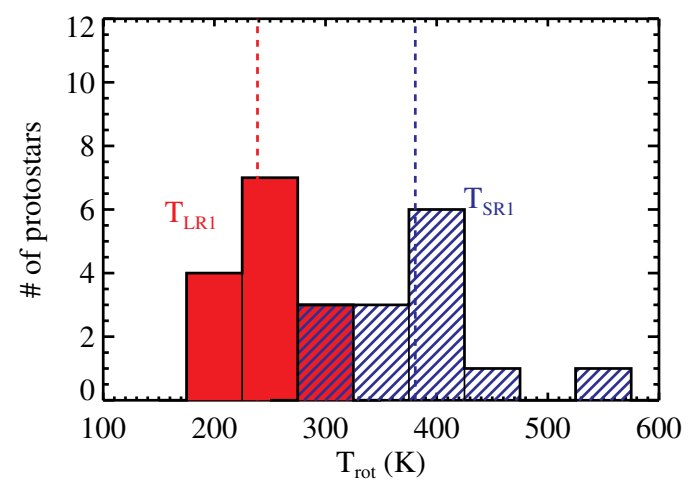

Figure 9. Distribution of rotational temperatures $T_{\mathrm{LR} 1}$ (red solid bars) and $T_{\mathrm{SR} 1}$ (blue line bars). The dashed lines represent the median values of $T_{\mathrm{LR} 1}$ (red) and $T_{\mathrm{SR} 1}$ (blue).

(A color version of this figure is available in the online journal.)

lobe of a molecular outflow away from the exciting source. The PACS spectral map centered on HOPS 87 is shown in Figure 13. Another protostar, HOPS 88 (a.k.a. MMS 5; Chini et al. 1997), seen toward the northwestern edge of this field, is driving a bipolar outflow along the east-west direction (Williams et al. 2003; Takahashi et al. 2008). The red lobe of this outflow falls on the two spaxels east of the on-source spaxel where HOPS 

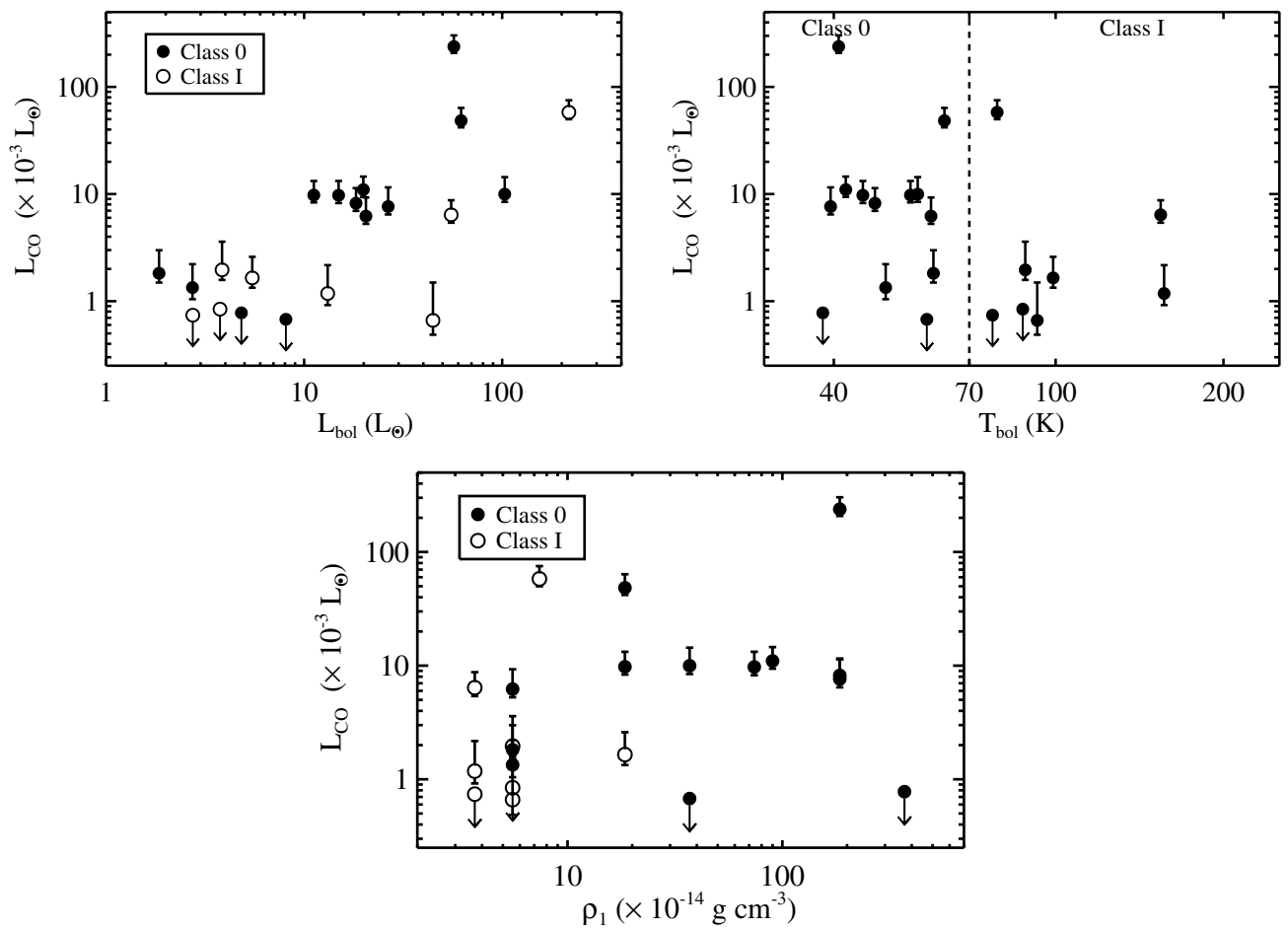

Figure 10. Total luminosity of the CO lines detected within the PACS wavelength range with $\mathrm{S} / \mathrm{N} \geqslant 3 \sigma$ as a function of $L_{\mathrm{bol}}, T_{\mathrm{bol}}$, and $\rho_{1}$. The lower error bars are measurement uncertainties. The upper error bars indicate the upper limits to $L_{\mathrm{CO}}$ obtained by adding the upper limits to the non-detected CO lines. Downward arrows indicate upper limits to $L_{\mathrm{CO}}$ for sources where no $\mathrm{CO}$ lines are detected in the PACS wavelength range.
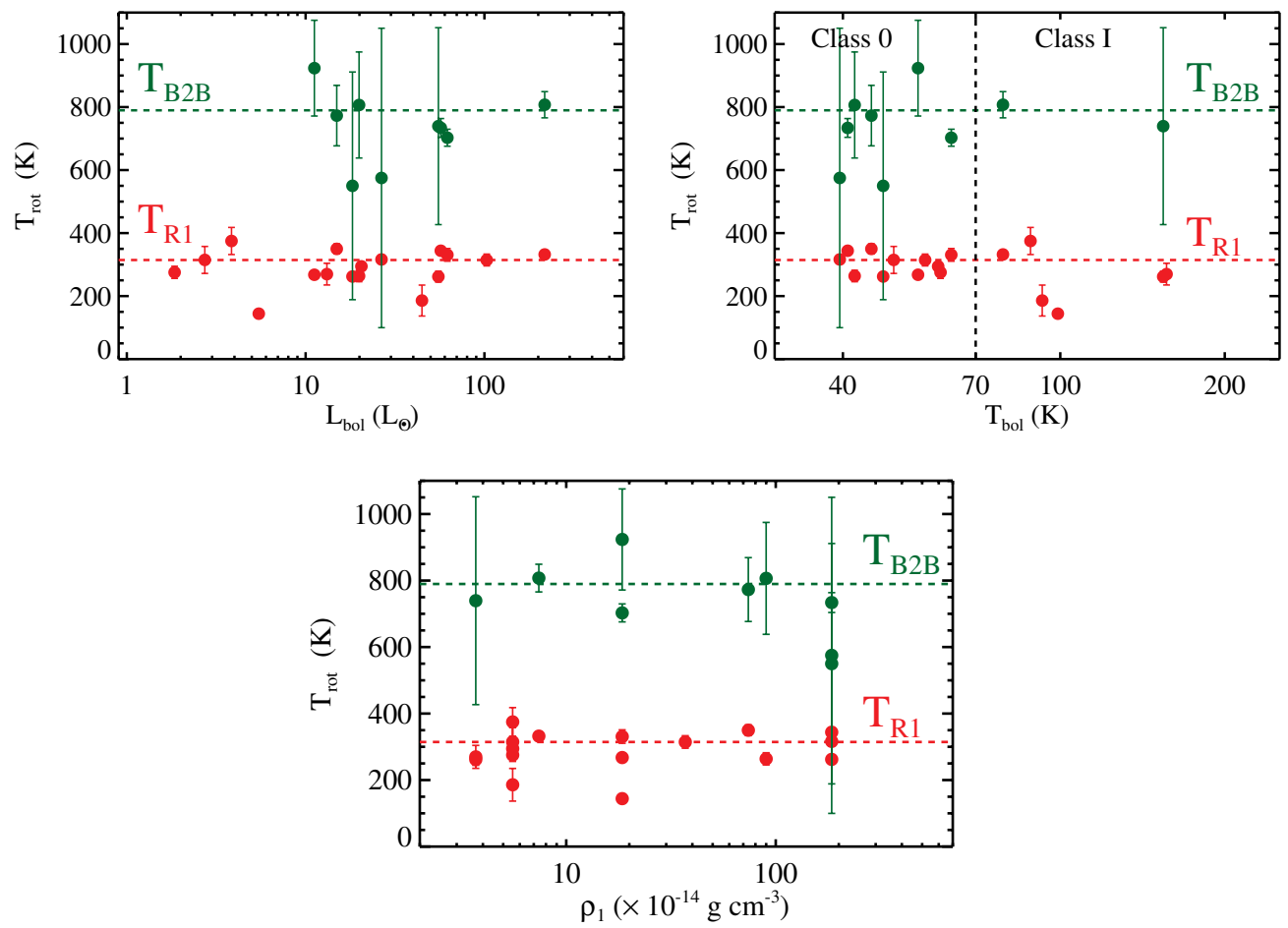

Figure 11. CO rotational temperatures $T_{\mathrm{R} 1}$ (red solid circles) and $T_{\mathrm{B} 2 \mathrm{~B}}$ (green solid circles) plotted against $L_{\mathrm{bol}}, T_{\mathrm{bol}}$, and $\rho_{1}$. The dashed lines represent the median values of $T_{\mathrm{R} 1}$ (red) and $T_{\mathrm{B} 2 \mathrm{~B}}($ green).

(A color version of this figure is available in the online journal.)

88 is located. Figure 13 shows that the line emission is brighter in the outflow spaxels labeled "off-source 1 and 2" than in the on-source spaxel. The CO rotational diagrams for the onsource spaxel and both off-source spaxels corresponding to the redshifted outflow lobe are shown in Figure 14. The rotational temperature, $T_{\mathrm{R} 1}$, measured for the on-source spaxel and for the outflow spaxels is nearly the same, within the uncertainties. The luminosities of the CO lines detected with PACS at the outflow lobe positions far away from the protostar are higher than that from the spaxel where HOPS 88 is located. For 


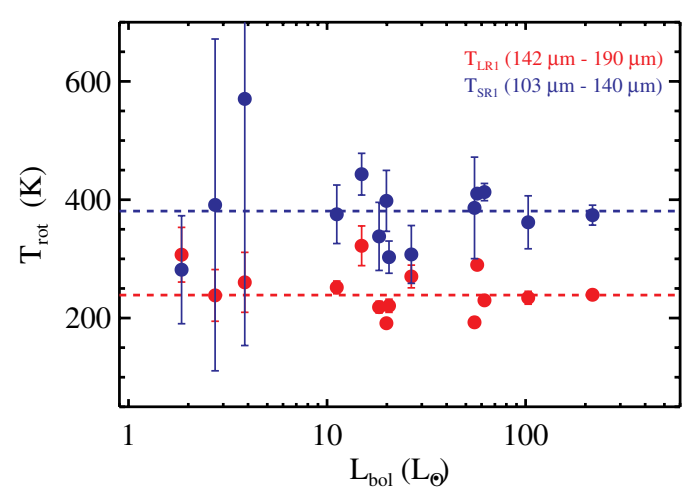

Figure 12. $T_{\mathrm{LR} 1}$ (red) and $T_{\mathrm{SR} 1}$ (blue) as functions of $L_{\mathrm{bol}}$.

(A color version of this figure is available in the online journal.)

example, $L_{\mathrm{CO}}$ from the off-source 2 position, which is $\sim 19^{\prime \prime}$ (projected distance $\sim 8000 \mathrm{AU}$ ) from the protostar, is about a factor of two higher than that for the on-source position (see Figure 14). Additionally, the rotational temperature, $T_{\mathrm{R} 1}$, and the CO luminosity, $L_{\mathrm{CO}}$, observed for the emission from the off-source positions are very similar to those found for the on-source positions of all the other protostars in our sample (see Figures 8 and 10).

\section{EXCITATION CONDITION OF THE CO EMITTING GAS}

Our most striking result is the independence of $\mathrm{CO}$ line ratios (or equivalently rotational temperatures) with respect to protostellar luminosity, and in a relatively large sample of protostars. The interpretation of this result and other apparent correlations, or lack thereof, between the observed properties of the $\mathrm{CO}$ emission and the protostellar properties depends on the physical conditions (temperature and density) in the emitting molecular gas. The $\mathrm{CO}$ emitting gas will be in LTE if the total gas (molecular hydrogen) density, $n\left(\mathrm{H}_{2}\right)$, is greater than $n_{\mathrm{cr}}$, the critical density of the $\mathrm{CO}$ transitions at a given temperature. The critical density of the lowest- $J$ transition ( $J=14-13)$ that we detect is $2-3 \times 10^{6} \mathrm{~cm}^{-3}$ and that of the highest- $J$ transition $(J=46-45)$ is $5-7 \times 10^{7} \mathrm{~cm}^{-3}$ for temperatures in the range of 300-3000 K (Neufeld 2012; Yang et al. 2010). Therefore, at densities $n\left(\mathrm{H}_{2}\right) \gtrsim 10^{8} \mathrm{~cm}^{-3}$, the CO rotational states will be thermalized and the observed rotational temperature is the physical temperature of the molecular gas. For gas densities $\lesssim 10^{6} \mathrm{~cm}^{-3}$, the CO excitation is sub-thermal ${ }^{15}$ and the rotational temperature can be significantly different from the physical temperature of the gas.

\subsection{Modeling of CO Emission from Protostars}

Recently, Neufeld (2012) has demonstrated that the observed curvature in the $\mathrm{CO}$ rotational diagrams obtained with Herschel/ PACS can be described surprisingly accurately by optically thin FIR CO emission originating in an isothermal medium or a medium with a power-law distribution of temperatures, both at uniform density. In the following, we use these sets of models to explore the likely physical conditions of the $\mathrm{CO}$ emitting gas in individual sources.

\footnotetext{
15 In this paper, as in previous papers in the literature, the excitation is said to be sub-thermal if the level populations of the upper states are smaller than the values that would be obtained in LTE; conversely, the excitation is described as thermal if the relative level populations are given by Boltzmann factors appropriate to the gas temperature. In either case, the process of collisional excitation involves colliding molecules that are assumed to have a thermal (i.e., Maxwell-Boltzmann) distribution of translational energies.
}

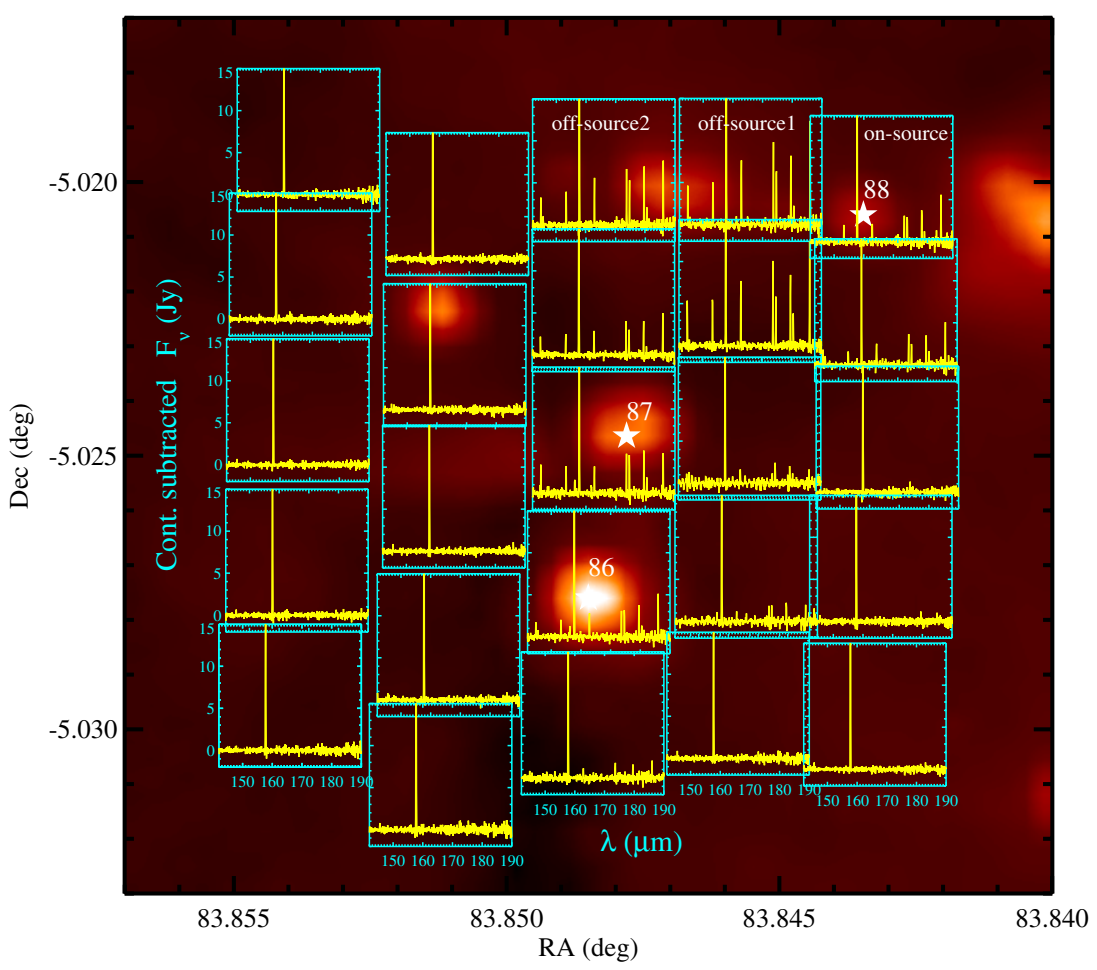

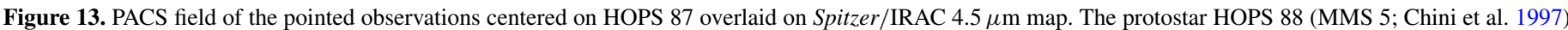

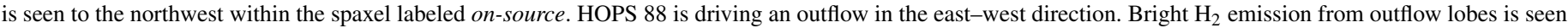

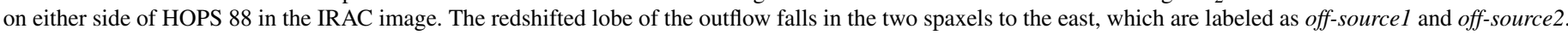
A continuum-subtracted spectrum in the spectral band Long R1 $(142-190 \mu \mathrm{m})$ is displayed in each spaxel.

(A color version of this figure is available in the online journal.) 

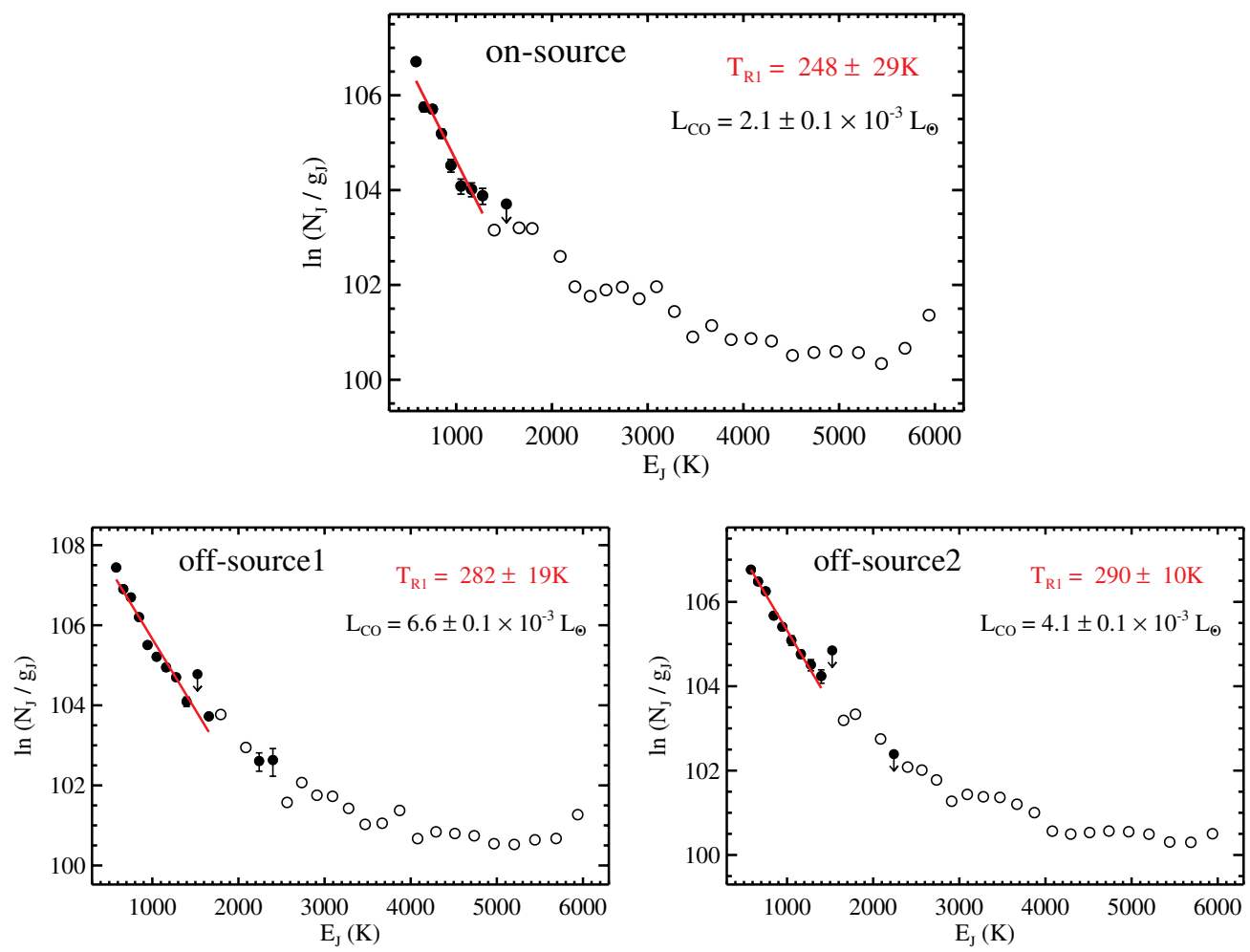

Figure 14. CO rotational diagrams for the on-source spaxel and the off-source outflow spaxels associated with HOPS 88 as shown in Figure 13.

(A color version of this figure is available in the online journal.)

Table 4

Best-fit Model Parameters

\begin{tabular}{|c|c|c|c|c|c|c|}
\hline \multirow[t]{2}{*}{ HOPS ID } & \multicolumn{3}{|c|}{ Isothermal } & \multicolumn{3}{|c|}{ Power Law } \\
\hline & $\begin{array}{c}T \\
(\mathrm{~K})\end{array}$ & $\begin{array}{c}n\left(\mathrm{H}_{2}\right) \\
\left(\times 10^{3} \mathrm{~cm}^{-3}\right)\end{array}$ & $\begin{array}{l}L_{\mathrm{CO}}{ }^{\mathrm{a}} \text { (Total) } \\
\left(\times 10^{-3} L_{\odot}\right)\end{array}$ & $\begin{array}{l}\text { Power Law } \\
\text { Index, } b\end{array}$ & $\begin{array}{c}n\left(\mathrm{H}_{2}\right) \\
\left(\times 10^{5} \mathrm{~cm}^{-3}\right)\end{array}$ & $\begin{array}{l}L_{\mathrm{CO}^{\mathrm{a}}} \text { (Total) } \\
\left(\times 10^{-3} L_{\odot}\right)\end{array}$ \\
\hline 56 & $2511_{-516}^{+306}$ & $1.6_{-1.4}^{+14.3}$ & 18 & $\ldots$ & $\ldots$ & $\ldots$ \\
\hline 60 & $2818_{-1405}^{+1162}$ & $0.2^{+62.9}$ & 14 & $\cdots$ & $\cdots$ & $\ldots$ \\
\hline 87 & $5011_{-1849}$ & $0.4_{-0.2}^{+24.7}$ & 17 & $\cdots$ & $\cdots$ & $\cdots$ \\
\hline 108 & $5011_{-545}$ & $15.8_{-5.8}^{+9.3}$ & 295 & $2.4_{-0.1}^{+0.1}$ & $6.3_{-2.3}^{+3.7}$ & 332 \\
\hline 182 & $3981_{-432}^{+485}$ & $1.6_{-1.4}^{+4.7}$ & 76 & $2.7_{-0.1}^{+0.1}$ & $6.3_{-2.3}^{+3.7}$ & 93 \\
\hline 203 & $5011_{-1030}$ & $15.8_{-5.8}^{+9.3}$ & 13 & $\ldots$ & $\cdots$ & $\cdots$ \\
\hline 288 & $3162_{-1167}^{+385}$ & $0.2^{+39.7}$ & 22 & $\ldots$ & $\ldots$ & $\ldots$ \\
\hline 310 & $3548_{-729}^{+918}$ & $6.3_{-6.2}^{+9.5}$ & 16 & $\ldots$ & $\ldots$ & $\ldots$ \\
\hline 368 & $2238_{-1238}^{+1309}$ & $2.5_{-2.4}^{+60.6}$ & 13 & $\ldots$ & $\ldots$ & $\ldots$ \\
\hline 369 & $2238_{-979}^{+923}$ & $2.5_{-2.4}^{+37.3}$ & 24 & $\ldots$ & $\ldots$ & $\cdots$ \\
\hline 370 & $4466_{-485}^{+545}$ & $1.0_{-0.8}^{+5.3}$ & 84 & $2.7_{-0.1}^{+0.1}$ & $10.0_{-3.7}^{+5.8}$ & 102 \\
\hline
\end{tabular}

Note. ${ }^{\text {a }} L_{\mathrm{CO}}$ listed here is the total $\mathrm{CO}$ luminosity derived from best-fit models for rotational transitions ranging from $J=1-0$ up to $J=80-79$.

\subsubsection{Isothermal Medium}

We first consider the simple case of an optically thin medium of uniform temperature and density. We computed the synthetic $\mathrm{CO}$ rotational diagrams for such a medium for a large range of values of $T, n\left(\mathrm{H}_{2}\right)$, and a column density parameter $\tilde{N}(\mathrm{CO})$ (for details, see Neufeld 2012). We then compared the model rotational diagrams with the observed ones to find the best-fit solutions by minimizing the reduced $-\chi^{2}$.

The best-fit models and physical parameters (corresponding to the minimum in reduced $-\chi^{2}$ ) obtained for $\mathrm{CO}$ emitting gas for all the protostars are shown in Figure 15. We only modeled 16 sources for which more than 3 CO lines are detected. The best-fit values of $T$ and $n\left(\mathrm{H}_{2}\right)$ are shown as a function of $L_{\text {bol }}$ in Figure 16. For five sources for which less than eight $\mathrm{CO}$ lines were detected, the density and temperature of the emitting medium are not constrained by the models. For all the other protostars, these parameters are well constrained; they are listed in Table 4. The optimal solutions have gas temperatures $T \gtrsim 2000 \mathrm{~K}$ and densities $n\left(\mathrm{H}_{2}\right) \lesssim 10^{4.5} \mathrm{~cm}^{-3}$. While gas temperatures are well constrained for these sources, for many of them only an upper limit could be obtained for densities. Also, 

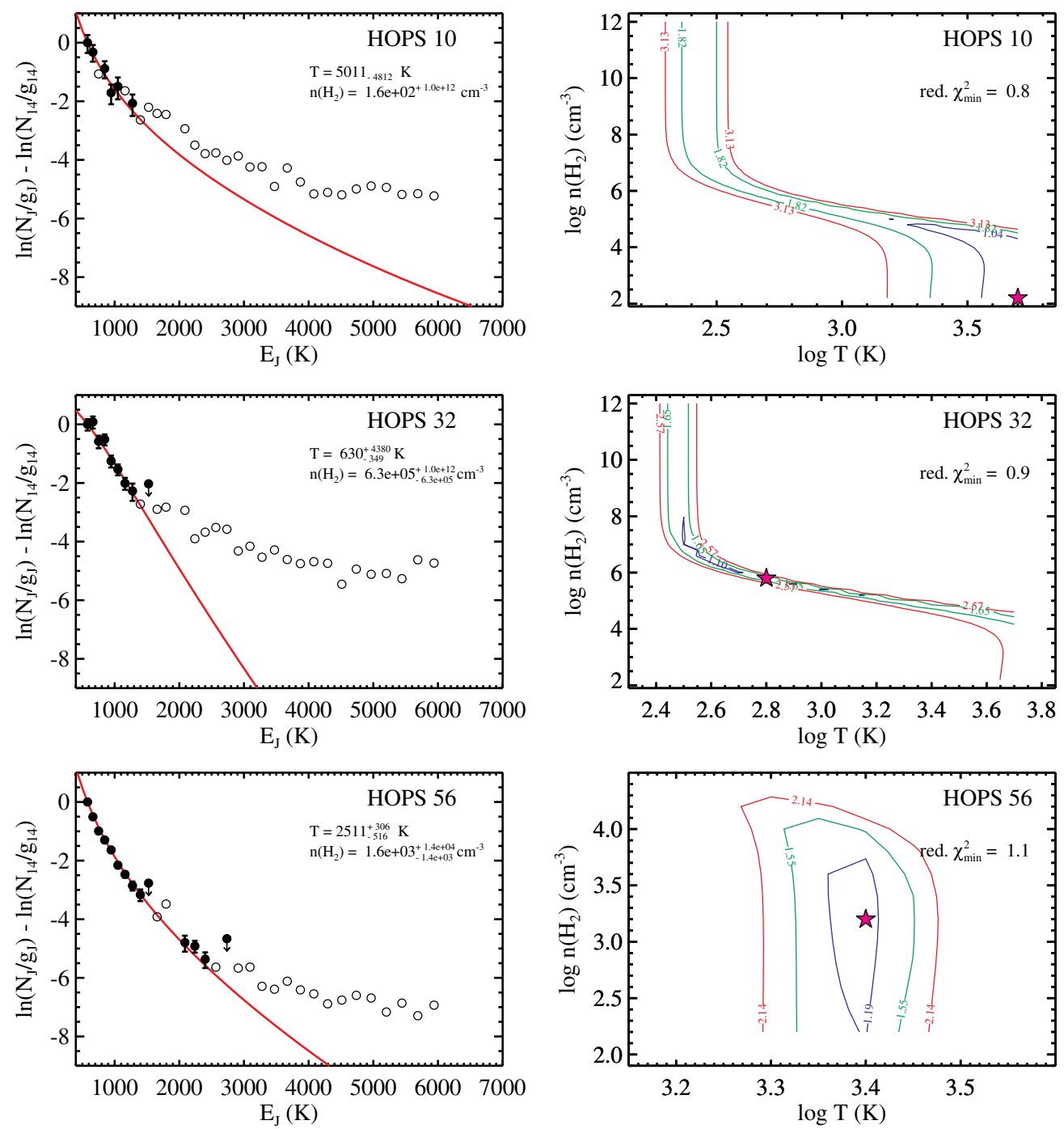

Figure 15. Left: rotational diagrams of the observed $\mathrm{CO}$ emission with the best fits for the isothermal medium model overlaid (solid red line). Downward arrows indicate upper limits to the fluxes of the $\mathrm{CO}$ lines which are blended with a nearby line. Open circles correspond to $3 \sigma$ upper limits for the non-detections. Right: reduced- $\chi^{2}$ contours for $T$ and $n\left(\mathrm{H}_{2}\right)$. The star symbol marks the minimum value of the reduced- $\chi^{2}$. The contours corresponding to $68.3 \%$ (blue), $95.4 \%$ (green), and $99.7 \%$ (red) confidence levels are shown.

(A color version of this figure is available in the online journal.)

several of them have the best-fit densities well below $10^{4} \mathrm{~cm}^{-3}$. This is because at high temperatures $(T \gtrsim 2000-3000 \mathrm{~K})$ and low densities $\left(n\left(\mathrm{H}_{2}\right) \lesssim 10^{4.5} \mathrm{~cm}^{-3}\right)$, the $\mathrm{CO}$ line ratios (or rotational diagrams) are insensitive to density; at these high gas temperatures the rotational diagrams are indistinguishable for densities below $10^{4.5} \mathrm{~cm}^{-3}$ (Neufeld 2012). Thus, our modeling shows that if the observed $\mathrm{CO}$ emission originates from a single isothermal component, then the $\mathrm{CO}$ excitation is sub-thermal.

For sources where the density and temperature of the $\mathrm{CO}$ emitting medium are well constrained, the total $\mathrm{CO}$ luminosities (for transitions $J=1-0$ up to $J=80-79$ ) obtained from the best-fit models range from $3.0 \times 10^{-1} L_{\odot}$ for HOPS 108 to $1.3 \times$ $10^{-2} L_{\odot}$ for HOPS 368 and 203 (see Table 4). The observed $L_{\mathrm{CO}}$ (see Table 3 and Figure 10) computed from the FIR CO lines detected with PACS ( $J=14-13$ up to $J=46-45$ ) is found to be $\sim 44 \%-81 \%$ of the total CO luminosity. Thus, if the CO emission from protostars is dominated by hot gas $(T \gtrsim 2000-3000 \mathrm{~K})$ at low densities $\left(n\left(\mathrm{H}_{2}\right) \lesssim 10^{4.5} \mathrm{~cm}^{-3}\right)$, most of the CO luminosity is emitted within the PACS wavelength range. The low-density, high-temperature solutions obtained from the fits to single- component isothermal models do not appear to be driven by the high- $J$ CO lines. We modeled rotational diagrams for $\mathrm{CO}$ lines observed in the R1 spectral band ( $\left.J_{\mathrm{up}}=14-25\right)$ separately, and found that, for most sources, a single-temperature component can explain the observed $\mathrm{CO}$ emission in the $\mathrm{R} 1$ band only for densities $n\left(\mathrm{H}_{2}\right)<10^{5} \mathrm{~cm}^{-3}$ and temperatures $T \gtrsim 2000 \mathrm{~K}$.

For five sources-HOPS 10, 32, 68, 85, and 343-where fewer than eight $\mathrm{CO}$ lines were detected $\left(J_{\text {up }} \leqslant 21\right)$, the densities are not constrained by the isothermal models. These objects are among the lowest $L_{\text {bol }}$ sources in our sample (see Figure 16). Although high-density, low-temperature LTE solutions cannot be ruled out for these sources, they are also consistent with the low-density $\left(n\left(\mathrm{H}_{2}\right)<10^{5} \mathrm{~cm}^{-3}\right)$, high-temperature $(T \gtrsim$ $2000 \mathrm{~K}$ ) solutions in the context of single component isothermal models.

Two sources in which several high- $J$ CO lines are detected (HOPS 182 and 370), the highest- $J$ lines $\left(J_{\text {up }} \geqslant 38\right.$ ) are not well fit by the single isothermal component models. This difficulty was noted by Neufeld (2012), who pointed out that CO rotational diagrams with large positive curvatures cannot be reproduced 

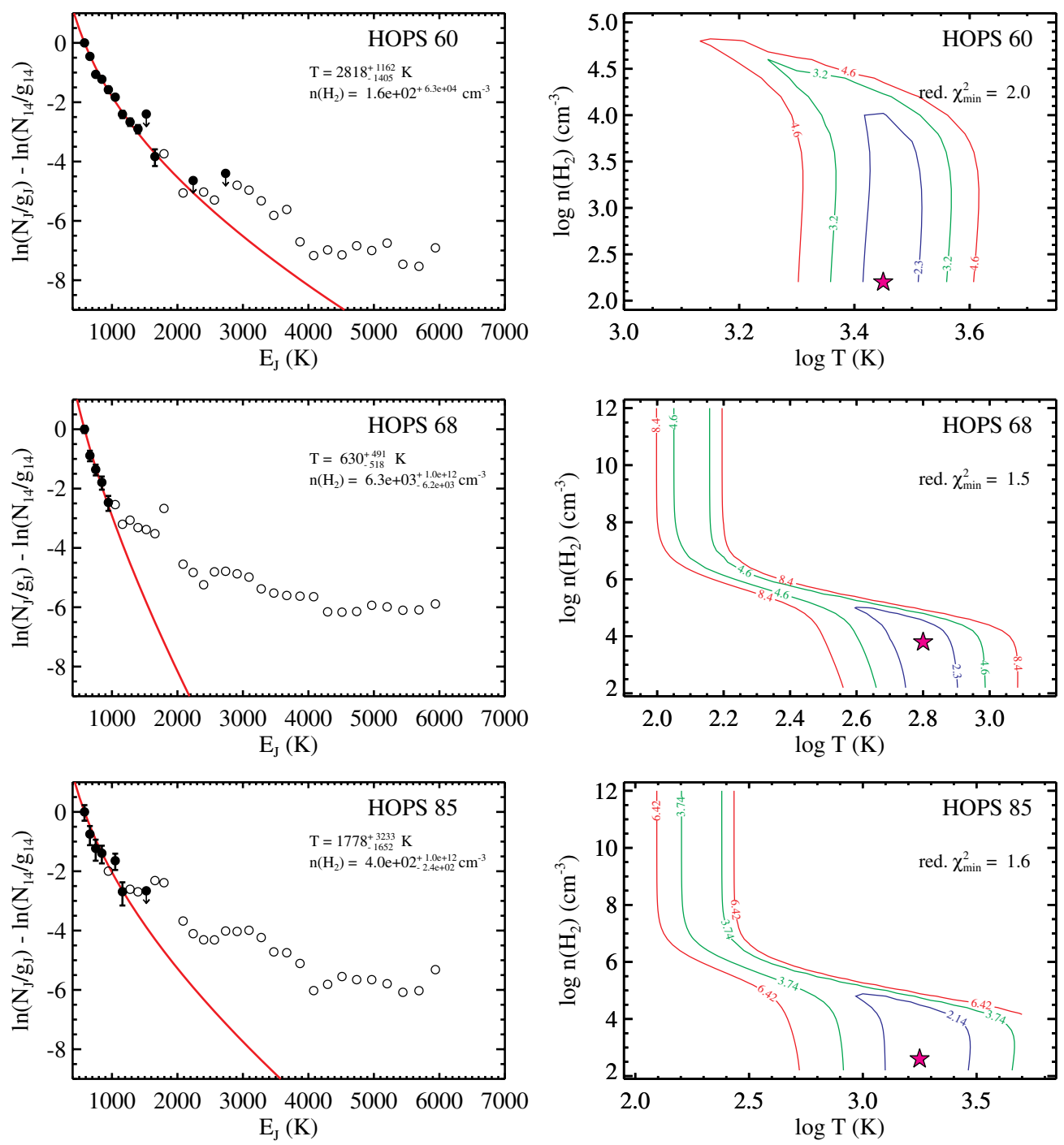

Figure 15. (Continued)

by a single-temperature component model; instead, these can be modeled using a medium with a power-law distribution of gas temperatures.

\subsubsection{Medium with a Power-law Distribution of Gas Temperatures}

Next, we model the observed $\mathrm{CO}$ emission as arising from an optically thin medium with a continuous distribution of gas temperatures to account for the presence of an admixture of gas temperatures along the line of sight. Following the approach of Neufeld (2012), the gas temperature distribution is approximated as a power law in these models: the column density of the medium in the temperature interval between $T$ and $T+d T$ has the form $d N \propto T^{-b} d T$, over a temperature range of $10-5000 \mathrm{~K}$ (for details see Neufeld 2012). Such power-law temperature distribution models have been successful in describing the observed rotational diagrams of molecular hydrogen (Neufeld \& Yuan 2008; Yuan \& Neufeld 2011; Giannini et al. 2011). We computed the synthetic rotational diagrams for $\mathrm{CO}$ emission from such a medium for a large range in density $n\left(\mathrm{H}_{2}\right)$, the power-law index $b$, and the column density parameter $\tilde{N}(\mathrm{CO})$ (for details, see Neufeld 2012). The best-fit solutions were obtained by minimizing the reduced $-\chi^{2}$.
The power-law temperature models constrain the density of the emitting gas only for three sources, HOPS 108, 182, and 370 , where more than $25 \mathrm{CO}$ lines are detected. These models are shown in Figure 17 and the best-fit values are listed in Table 4. For HOPS 108, whose rotational diagram has only a modest positive curvature, the power-law fit is only marginally better (in terms of reduced- $\chi^{2}$ ) than the isothermal fit. The rotational diagrams of HOPS 182 and 370 have larger positive curvature and for these two sources, power-law temperature models provide significantly better fits (in terms of reduced$\chi^{2}$ ) than those obtained from a single-temperature component model. For these three sources, the density of the emitting medium is tightly constrained between $4 \times 10^{5} \mathrm{~cm}^{-3}$ and $10^{6} \mathrm{~cm}^{-3}$, indicating that the excitation of $\mathrm{CO}$ is not thermal. The total CO luminosity derived from the best-fit power-law solutions (for transitions with $J_{\text {up }}=1$ to 80 ) is slightly higher than that obtained from the isothermal model fits for HOPS 108, 182, and 370 (see Table 4). Comparison with the observed $L_{\mathrm{CO}}$ (Table 3 ) shows that $52 \%-72 \%$ of the total CO luminosity in these objects is emitted within the PACS wavelength range.

For all the other sources with fewer number of detected CO lines, gas densities are not well constrained. Acceptable solutions could be found for high densities $\left(n\left(\mathrm{H}_{2}\right)>10^{6} \mathrm{~cm}^{-3}\right)$ 

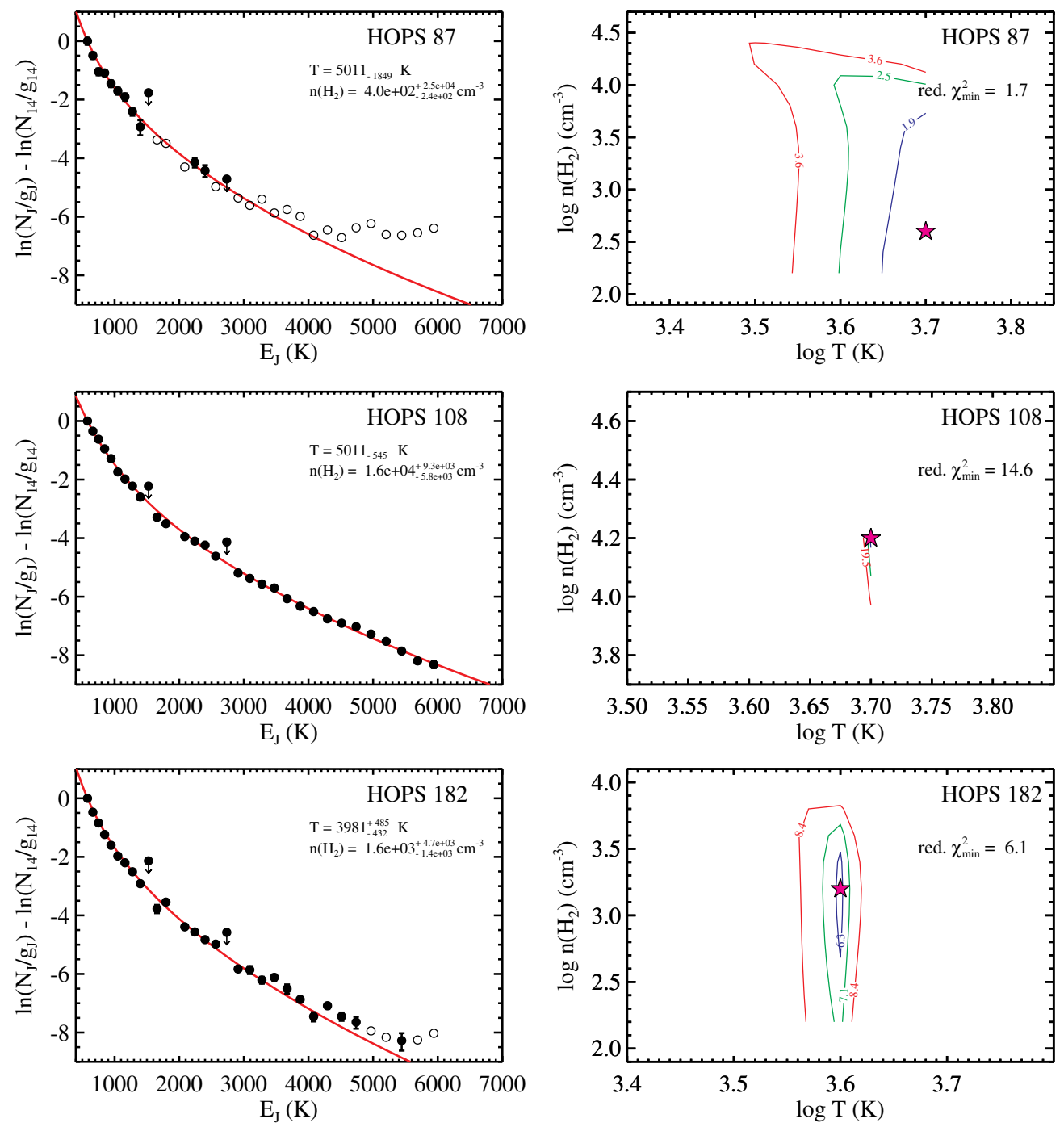

Figure 15. (Continued)

with steeper power-law indices $(b>3)$ and for low densities $\left(n\left(\mathrm{H}_{2}\right)<10^{5} \mathrm{~cm}^{-3}\right)$ with $b<2$. The low-density solutions with $b \ll 2$ approach the isothermal solutions described in the previous section. The degeneracies in the multi-component temperature fits are primarily because fewer $\mathrm{CO}$ lines are detected in these sources. When more lines are detected, the models tightly constrain the physical parameters of the emitting medium, as in the cases of HOPS 108, 182, and 370. Nevertheless, the ranges of density and power-law index found for these three sources also appear to be consistent with the observed $\mathrm{CO}$ emission from most of the other sources in our sample. This is demonstrated in Figure 18. Power-law temperature models with $b$ in the range of 2.0-3.0 and densities in the range of $10^{5}-10^{6} \mathrm{~cm}^{-3}$ can explain the observed rotational diagrams of all the other sources for which fewer lines are detected.

\subsubsection{Degeneracies in the Model Fits}

There are significant degeneracies in the model fits. In the five sources where fewer than eight $\mathrm{CO}$ lines detected, neither models can rule out high-density $\left(n\left(\mathrm{H}_{2}\right) \gtrsim 10^{8} \mathrm{~cm}^{-3}\right)$ solutions where the emitting gas is in LTE. The more flexible power-law temperature models tightly constrain density to $10^{5}-10^{6} \mathrm{~cm}^{-3}$ only for the three sources where more than 25 lines are detected.
For other sources, even though the power-law models with densities in the range of $10^{5}-10^{6} \mathrm{~cm}^{-3}$ are consistent with the observed emission, we cannot rule out higher density solutions. Moreover, the power-law temperature models that we use are at uniform density. In reality, the $\mathrm{CO}$ emitting medium is likely to have a range of temperature and density. Models with multiple temperature and density components can possibly reproduce the observed CO emission for LTE conditions.

\subsection{Thermal versus Sub-thermal Excitation}

The "universality" of CO rotational temperatures (line ratios), and the degeneracies of the simple models, suggest strongly that the $\mathrm{CO}$ excitation is largely either thermal at low temperatures ( $\sim 300 \mathrm{~K}$ for the lines observed in the $R 1$ band) or subthermal at high temperatures $(\gtrsim 1000-2000 \mathrm{~K})$. If the CO excitation is thermal, i.e., $n\left(\mathrm{H}_{2}\right) \gtrsim 10^{8} \mathrm{~cm}^{-3}$, the rotational temperature should approach the physical temperature of the gas and is insensitive to density. This can be seen from Figure 19, where the average rotational temperatures predicted for the $\mathrm{CO}$ emission from an optically thin, isothermal medium are shown for various gas temperatures and densities (also see Neufeld 2012). At these high densities, the observed distributions of $T_{\mathrm{R} 1}$ and $T_{\mathrm{B} 2 \mathrm{~B}}$ indicate a narrow range in the gas temperature for both the components (see Figure 19). This implies that, 

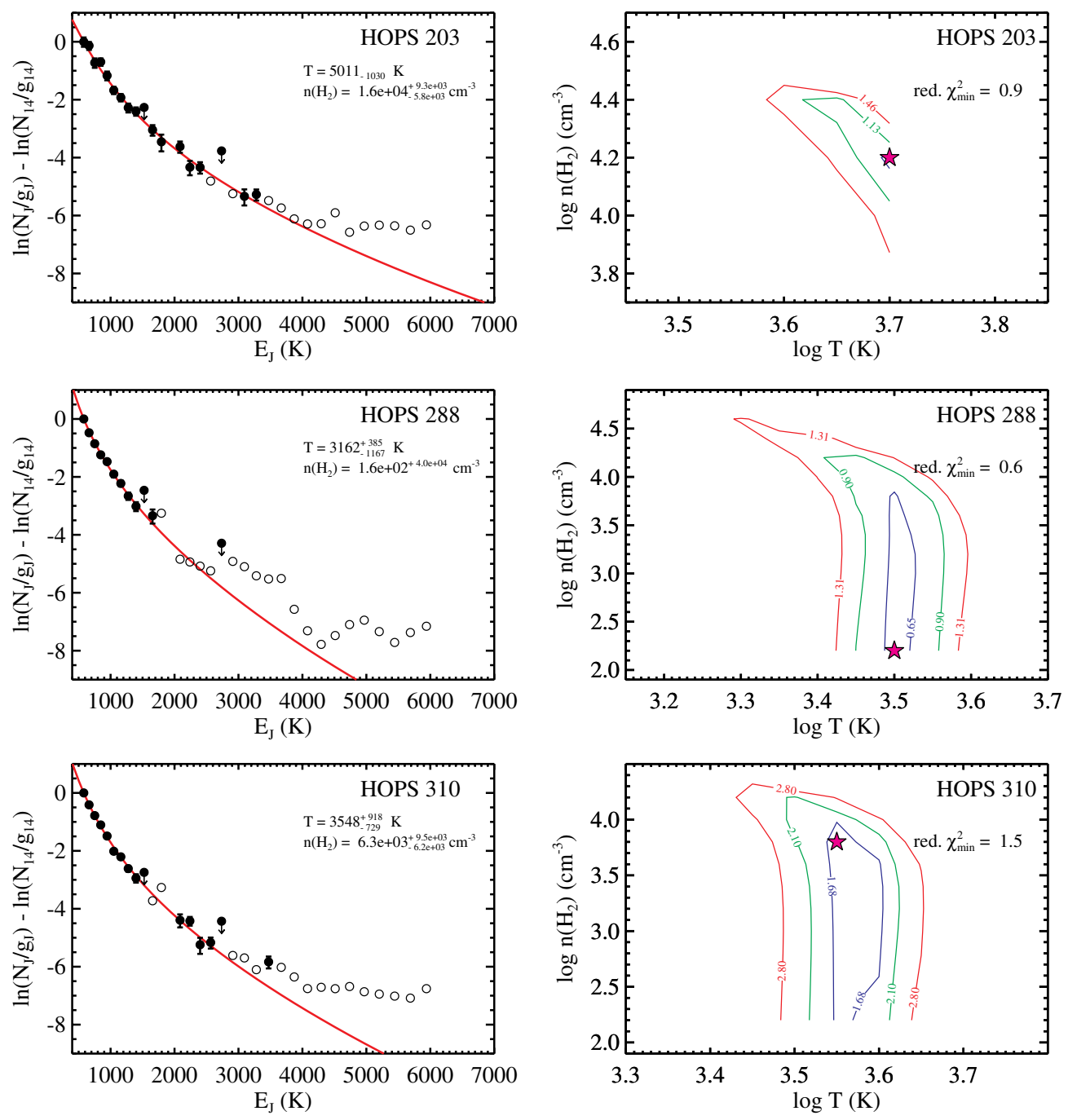

Figure 15. (Continued)

if the $\mathrm{CO}$ is thermally excited, the physical temperature of the emitting gas should be very similar for protostars whose luminosities differ by two orders of magnitude. Additionally, if the $\mathrm{CO}$ excitation is thermal, the observed positive curvature of the rotational diagrams indicates the presence of multiple temperature components. The average rotational temperatures, $T_{\mathrm{R} 1}, T_{\mathrm{B} 2 \mathrm{~B}}$, and $T_{\mathrm{B} 3 \mathrm{~A}}$ (see Table 3 ), are significantly different in each source, which suggests the presence of at least three temperature components. Moreover, the observed distribution of the rotational temperatures $T_{\mathrm{LR} 1}$ and $T_{\mathrm{SR} 1}$ (see Section 4.1) indicates that even the $\mathrm{CO}$ lines in the $\mathrm{R} 1$ spectral band $\left(J_{\text {up }}=\right.$ 14-25) require at least two temperature components if the excitation is thermal. Thus, for most sources in our sample, four or more components would be required to explain the observed emission if the emitting gas is in LTE. The narrow range in the rotational temperatures observed for the protostars in our sample then requires that these multiple temperature components of the $\mathrm{CO}$ emitting gas remain nearly identical for sources with $L_{\text {bol }}$ in the range of $\sim 2-217 L_{\odot}$. Thus, the primary weakness of LTE solutions is that they require heating mechanisms that maintain relatively constant temperatures in multiple gas components over a range of protostellar luminosity spanning two orders of magnitude.

However, if the $\mathrm{CO}$ excitation is sub-thermal, gas at high temperatures $(T \gtrsim 1000-2000 \mathrm{~K})$ and moderate densities
$\left(n\left(\mathrm{H}_{2}\right) \lesssim 10^{6} \mathrm{~cm}^{-3}\right)$ can reproduce the observed $\mathrm{CO}$ emission from protostars. For low densities, $n\left(\mathrm{H}_{2}\right) \lesssim 10^{4.5} \mathrm{~cm}^{-3}$, the $\mathrm{CO}$ rotational diagrams are well fit by emission from an isothermal medium with temperatures in the range of 2000-5000 K. One attractive feature of this set of solutions is that in the low-density limit, the rotational temperatures are insensitive to density and depends only on the temperature (Neufeld 2012; also see Figure 19). At densities below $10^{4.5} \mathrm{~cm}^{-3}$, a large range in the gas temperatures results only in a narrow range in rotational temperatures. For example, emitting gas at temperatures in the range of 500-5000 K can reproduce the observed range in $T_{\mathrm{R} 1}$, as can be seen from Figure 19. In this case, it is somewhat easier to explain the observed behavior of rotational temperatures as a function of $L_{\mathrm{bol}}$. In protostars with higher $L_{\mathrm{bol}}$, the CO emitting gas is possibly hotter, but the resulting rotational temperatures are not very different from those produced by cooler $\mathrm{CO}$ gas in low $L_{\text {bol }}$ sources. Note, however, that the isothermal solutions are only illustrative and multiple temperature components must be present; sources that show large positive curvature in their rotational diagrams (HOPS 182 and 370$)$, the high- $J\left(J_{\text {up }} \gtrsim 38\right)$ lines are not well fit by a single-temperature component.

Alternatively, we can model the temperature of the emitting medium as a continuous power-law distribution where the parameters are now the power-law index and the density of the gas, as has been done successfully for the excitation $\mathrm{H}_{2}$ 

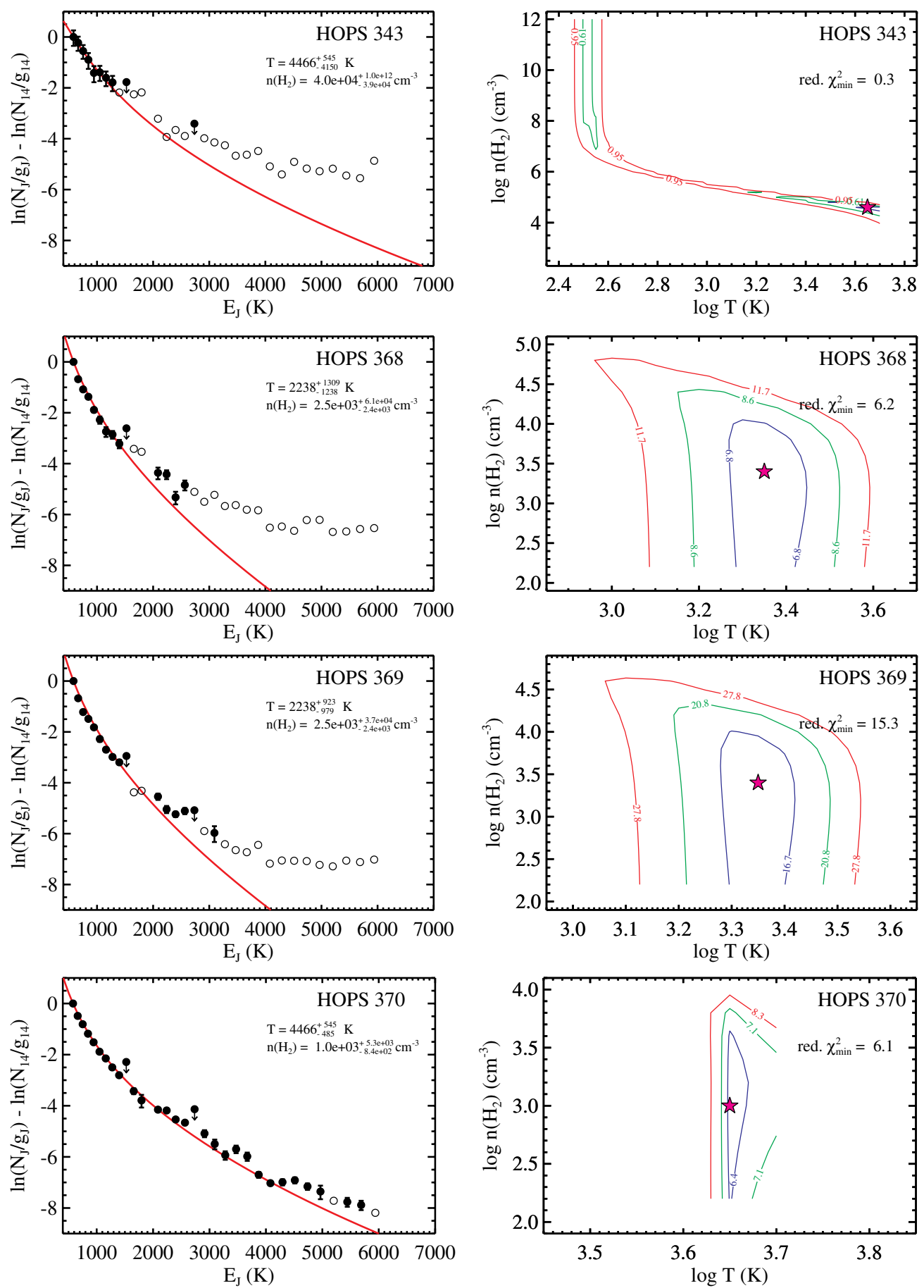

Figure 15. (Continued)

(Neufeld \& Yuan 2008; Yuan \& Neufeld 2011; Giannini et al. 2011). The observed line ratios of all the sources are consistent with power-law models with power-law index, $b=2.0-3.0$ and with densities in the range of $10^{5}-10^{6} \mathrm{~cm}^{-3}$. These densities are also below the critical densities of the observed $\mathrm{CO}$ transitions and the excitation is sub-thermal. Even when multiple gas components with a power-law distribution of temperatures are present, a range of excitation conditions $\left(n\left(\mathrm{H}_{2}\right)\right.$ and $\left.b\right)$ can explain the narrow range in the observed rotational temperatures as long as the excitation is sub-thermal. Additionally, the $\mathrm{CO}$ emission observed with PACS is dominated by the hightemperature $(T \gtrsim 1000 \mathrm{~K})$ components, even when lower temperature components are present. For the best-fit model for HOPS $108\left(n\left(\mathrm{H}_{2}\right)=6.0 \times 10^{5} \mathrm{~cm}^{-3}\right.$ and $\left.b=2.4\right)$, the gas components with $T \gtrsim 1000 \mathrm{~K}$ dominates the emission even in the R1 spectral band for CO transitions $J_{\text {up }} \geqslant 18$, and components with $T \gtrsim 2000 \mathrm{~K}$ contributes more than $50 \%$ of the observed flux for lines $\left(J_{\text {up }} \geqslant 27\right)$ observed in B2B and B3A spectral bands.

In reality, a solution intermediate to those obtained with the power-law and isothermal models, with a range of temperatures in which the lower temperature $(T \lesssim 1000 \mathrm{~K})$ components do not contribute significantly to the observed emission is likely to be more robust. Thus, if the FIR CO emission observed with 


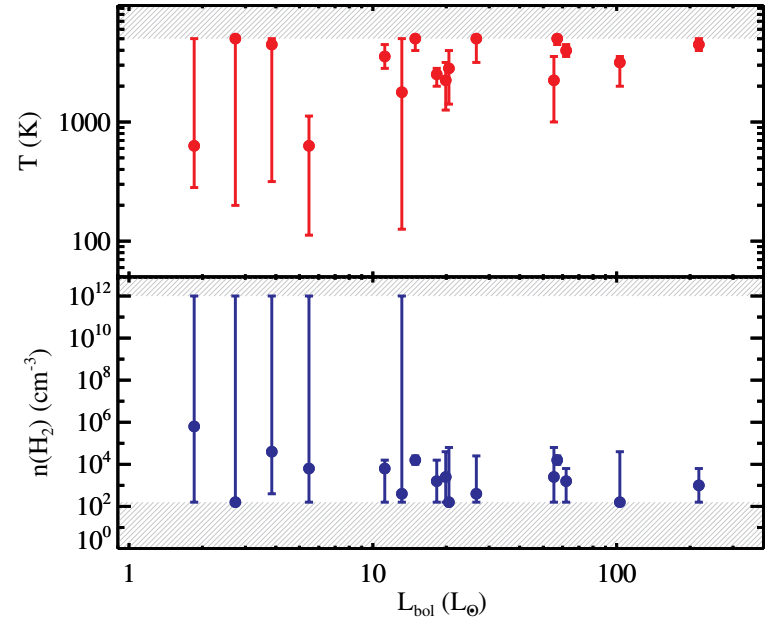

Figure 16. Best-fit values of $T$ and $n\left(\mathrm{H}_{2}\right)$ for $\mathrm{CO}$ emission from an isothermal medium plotted against $L_{\mathrm{bol}}$. The uncertainties shown correspond to $99.7 \%$ confidence interval. The gray shaded regions indicate $T$ and $n\left(\mathrm{H}_{2}\right)$ parameter space not explored by our models.

(A color version of this figure is available in the online journal.)
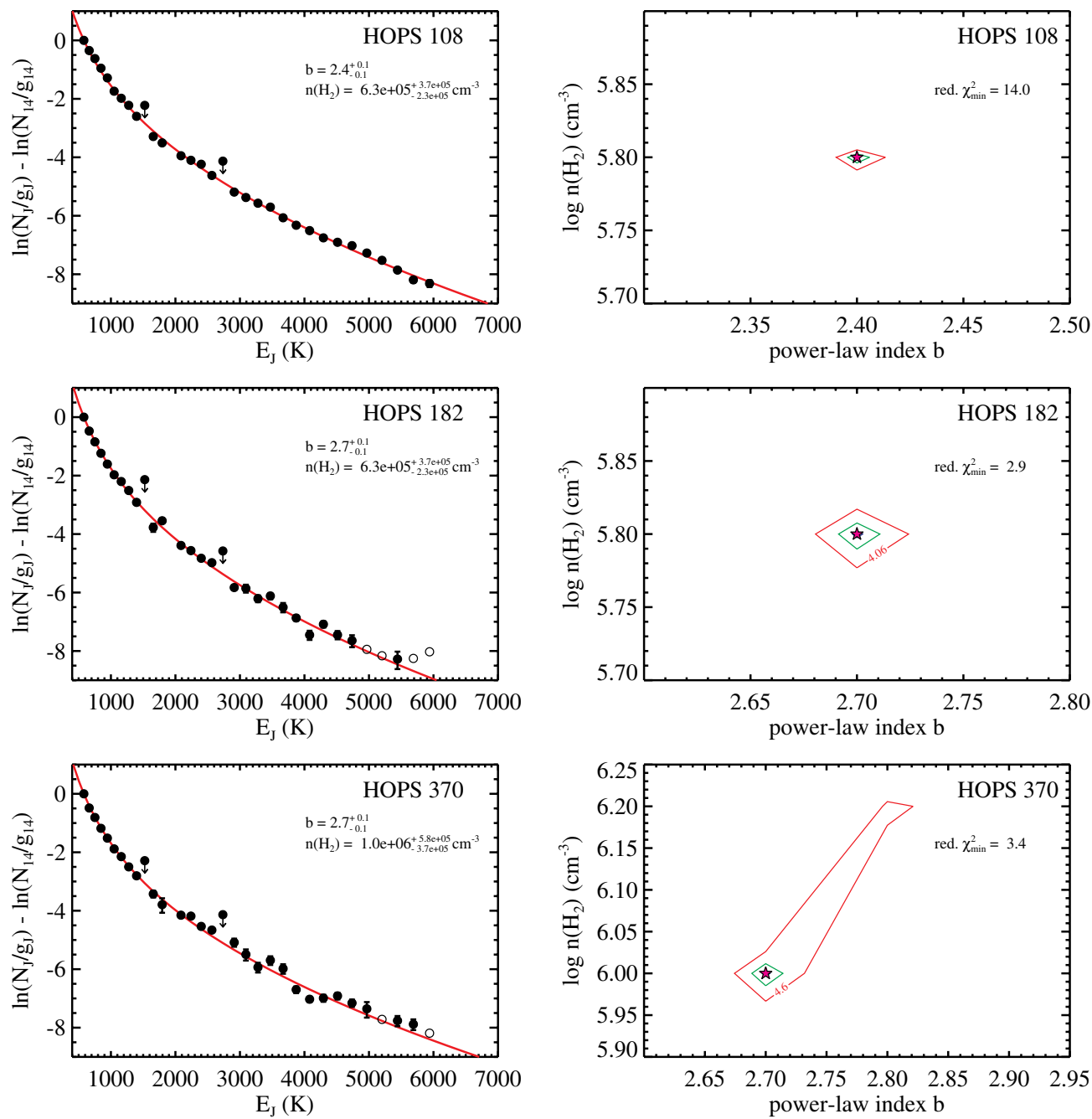

Figure 17. Left: rotational diagrams of the observed $\mathrm{CO}$ emission with the best fits for the power-law temperature model overlaid (solid red line). Downward arrows indicate upper limits to the fluxes of the $\mathrm{CO}$ lines which are blended with a nearby line. Open circles correspond to $3 \sigma$ upper limits for the non-detections. Right: reduced- $\chi^{2}$ contours for $T$ and $n\left(\mathrm{H}_{2}\right)$. The star symbol marks the minimum value of the reduced- $\chi^{2}$. The contours corresponding to $68.3 \%$ (blue), $95.4 \%$ (green), and $99.7 \%$ (red) confidence levels are shown.

(A color version of this figure is available in the online journal.) 

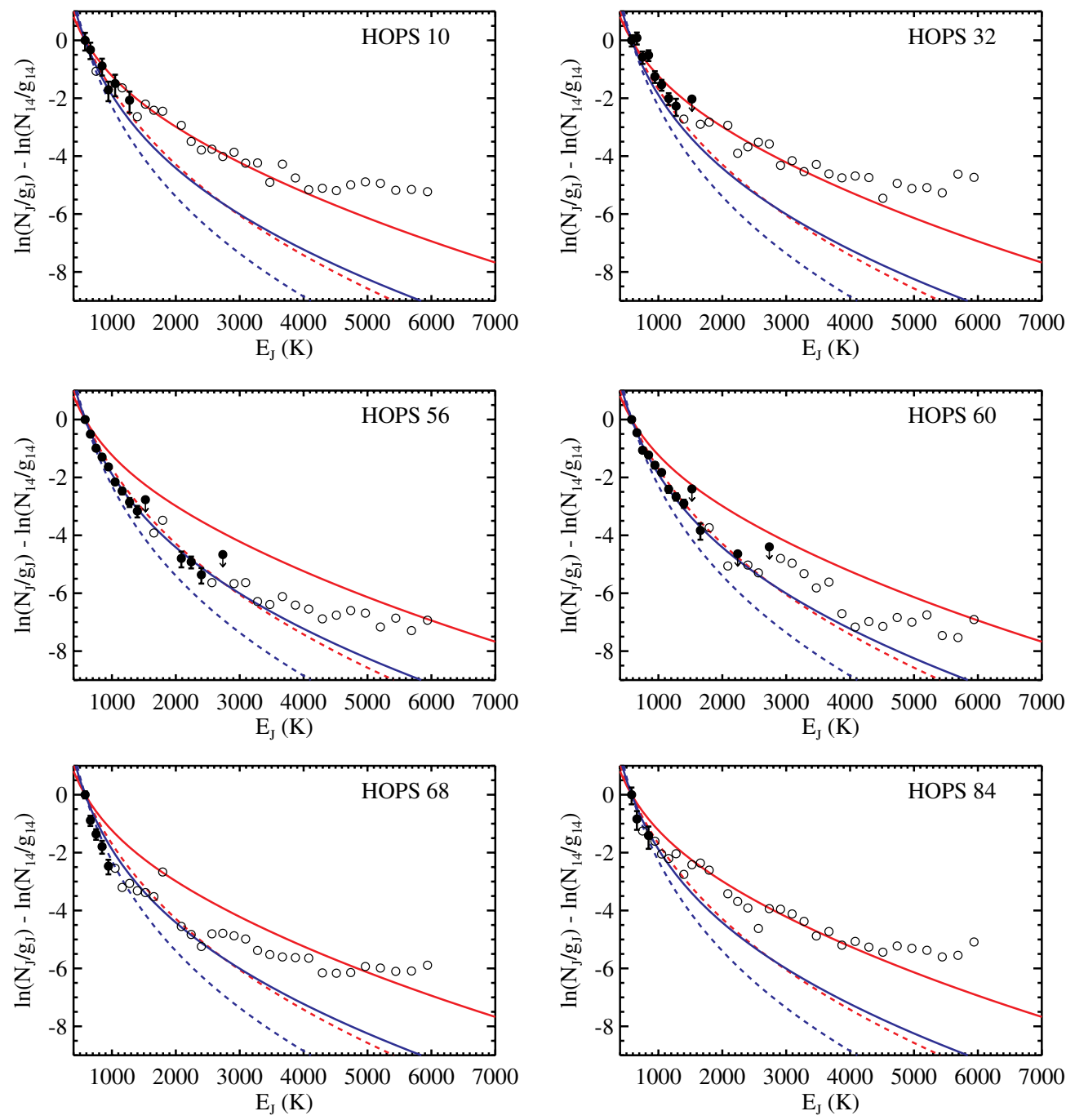

Figure 18. Synthetic rotational diagrams for a medium with a power-law gas temperature distribution with $b=3.0$ (blue) and $b=2.0$ (red) for densities $n\left(\mathrm{H}_{2}\right)=10^{5} \mathrm{~cm}^{-3}$ (dashed line) and $n\left(\mathrm{H}_{2}\right)=10^{6} \mathrm{~cm}^{-3}$ (solid line) overlaid on the observed rotational diagrams. Downward arrows indicate upper limits to the fluxes of the $\mathrm{CO}$ lines which are blended with a nearby line. Open circles correspond to $3 \sigma$ upper limits for the non-detections.

(A color version of this figure is available in the online journal.)

cavity walls (van Kempen et al. 2010b; Fich et al. 2010; Visser et al. 2012). The primary motivation for invoking the PDRs has been the narrow $\left(\mathrm{FWHM} \lesssim 2 \mathrm{~km} \mathrm{~s}^{-1}\right.$ ) velocity component seen in the spectrally resolved line profiles of the low- $J\left(J_{\text {up }} \leqslant 10\right)$ $\mathrm{CO}$ lines observed toward low-mass protostars (van Kempen et al. 2009a, 2009b; van Dishoeck et al. 2009; Y1ldı et al. 2010, 2012). Since PACS lines are spectrally unresolved, it was assumed that this narrow component due to UV-heated cavity walls also contributes to the line flux of the higher$J\left(J_{\text {up }} \geqslant 14\right)$ CO transitions observed with PACS. Both the UV-heated and shock-heated gas components were found to be necessary to explain the observed $\mathrm{CO}$ emission in three particular protostars modeled in detail by Visser et al. (2012). In the following, we discuss how well these two mechanisms can explain the observed properties of the FIR CO emission from a larger sample of protostars. We argue that emission from the UV-heated PDRs along the cavity walls is unlikely to be the dominant component of the observed FIR CO emission. Most of the observed emission, instead, is likely to originate from lowdensity $\left(n\left(\mathrm{H}_{2}\right) \lesssim 10^{6} \mathrm{~cm}^{-3}\right)$ molecular gas, heated in outflow shocks, within $2000 \mathrm{AU}$ from the protostars.

\subsection{Origin in PDRs along Envelope Cavity Walls}

In PDR models, energy released from the mass accretion onto the protostar from the surrounding disk is the source of the UV flux which heats the cavity walls (Visser et al. 2012). The accretion luminosity of the protostars scales with $L_{\mathrm{bol}}$ (Kenyon \& Hartmann 1995; Evans et al. 2009) and therefore $L_{\mathrm{UV}}$ is likely to increase with increasing $L_{\mathrm{bol}}$. In the PDR models of Visser et al. (2012), most of the UV-excited emission originates at densities between $10^{6}$ and $10^{9} \mathrm{~cm}^{-3}$ and is therefore close to LTE. The total CO luminosity in this case is proportional to the total number of the $\mathrm{CO}$ molecules present (or equivalently mass of the $\mathrm{CO}$ gas) for a constant gas temperature. Thus, in protostars with higher $L_{\mathrm{bol}}$ (and therefore higher $L_{\mathrm{UV}}$ ), a larger amount of gas will be heated, in turn resulting in a higher $L_{\text {CO }}$. The PDR models can explain the observed correlation between $L_{\mathrm{CO}}$ and $L_{\mathrm{bol}}$, but only if the temperatures of the gas components remain the same in all the sources. Indeed, the various rotational temperatures computed $-T_{\mathrm{LR} 1}, T_{\mathrm{SR} 1}, T_{\mathrm{R} 1}$, and $T_{\mathrm{B} 2 \mathrm{~B}}$ - for the protostars in our sample are found to be within narrow ranges. If the $\mathrm{CO}$ excitation is thermal, as is the 

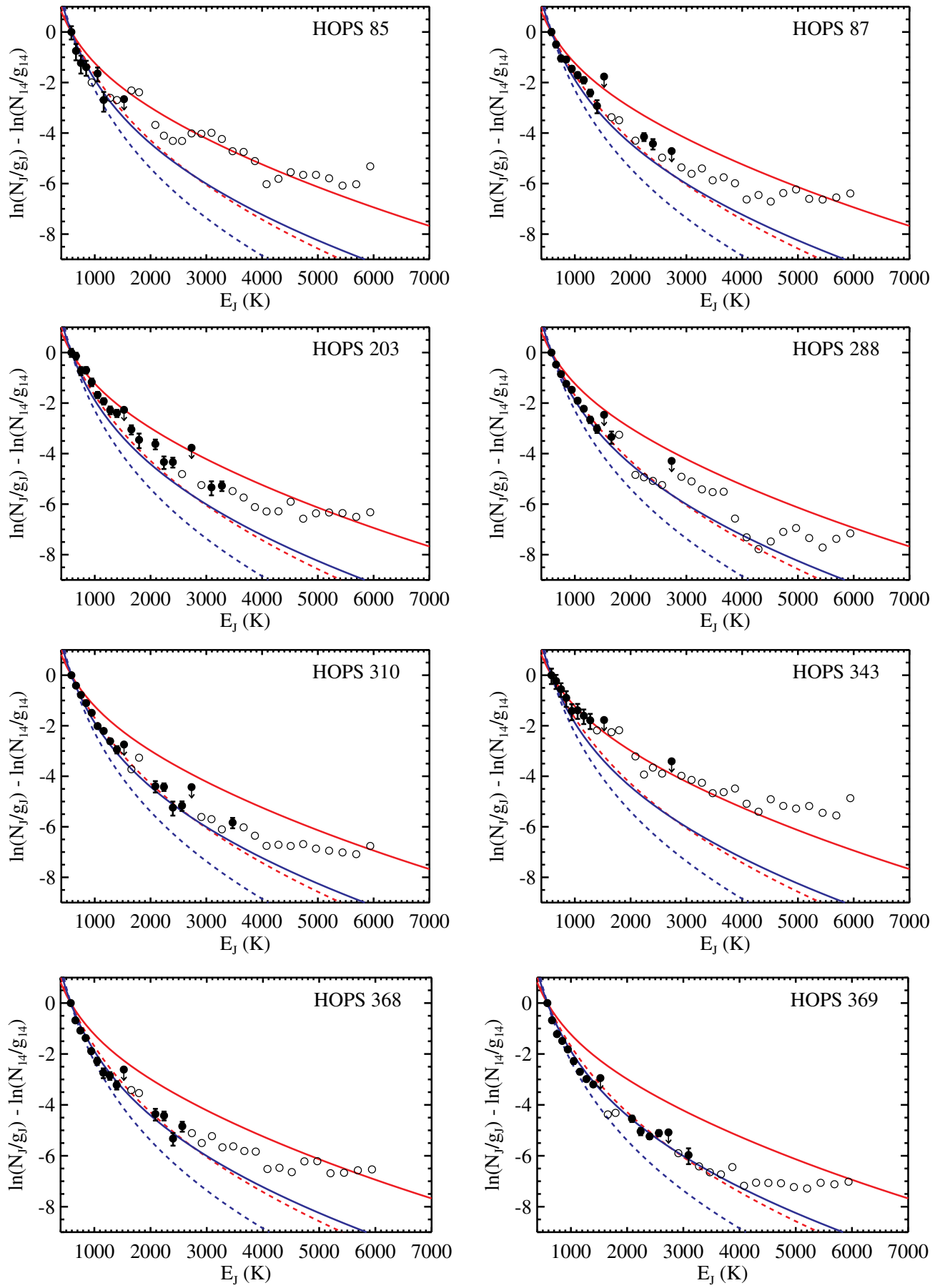

Figure 18. (Continued)

case in PDRs, the average rotational temperature computed for a small range in $E_{J}$ should be representative of the physical temperature of the gas component contributing to those $\mathrm{CO}$ lines. It is difficult, however, to obtain similar gas temperatures in PDRs along the envelope cavity walls for protostars with $L_{\text {bol }}$ ranging over two orders of magnitude. In PDRs, gas-grain collisions and the subsequent thermal emission from the dust grains is the dominant cooling mechanism for molecular gas densities $n\left(\mathrm{H}_{2}\right) \gtrsim 10^{6} \mathrm{~cm}^{-3}$, and the cooling rate is $\propto n\left(\mathrm{H}_{2}\right)^{2}$. Since the photoelectric heating rate is $\propto L_{\mathrm{UV}} n\left(\mathrm{H}_{2}\right)$, the gas temperature roughly scales as $T \propto L_{\mathrm{UV}} / n\left(\mathrm{H}_{2}\right)$. The $\mathrm{CO}$ gas temperature can remain nearly constant in protostars with widely ranging $L_{\text {bol }}$ values only if the envelope density scales linearly with $L_{\text {bol }}$. Figure 2, however, shows that the envelope density at $1 \mathrm{AU}, \rho_{1}$, is uncorrelated with $L_{\mathrm{bol}}$, so it is unlikely that the gas temperatures along the cavity walls are similar in all the protostars.

In Section 4.3, we showed that the luminosity of the observed $\mathrm{CO}$ emission from an outflow lobe $\sim 19^{\prime \prime}$ (projected distance $\sim 8000$ AU) away from the protostar is a factor of two higher than that observed for the on-source emission. If the UVradiation from the protostar were responsible for the heating of the gas, then hotter and more luminous $\mathrm{CO}$ emission would be expected from the on-source position, the opposite of what is observed. Moreover, the rotational temperature and luminosity of the $\mathrm{CO}$ emission from the outflow lobe far away from the 


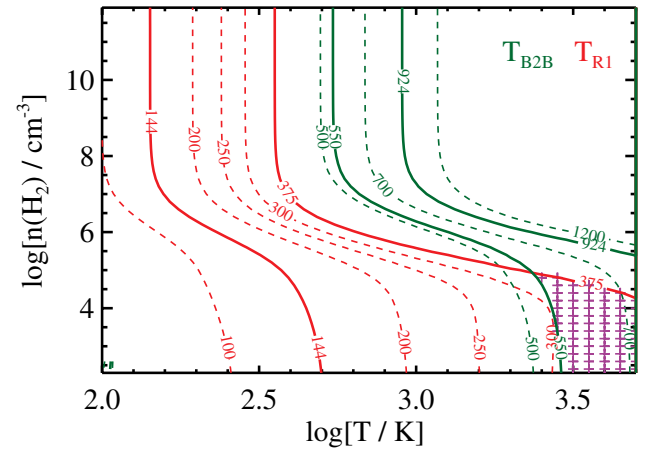

Figure 19. Synthetic rotational temperatures, $T_{\mathrm{R} 1}$ (red) and $T_{\mathrm{B} 2 \mathrm{~B}}$ (green), computed for $\mathrm{CO}$ emission from an optically thin, isothermal, uniform density medium (dashed contours). The solid contours represent the upper and lower bounds for the observed values of $T_{\mathrm{R} 1}$ (red) and $T_{\mathrm{B} 2 \mathrm{~B}}$ (green). The shaded region (purple crosses) indicates gas temperatures and densities for which a single-component model can simultaneously reproduce both the observed $T_{\mathrm{R} 1}$ and $T_{\mathrm{B} 2 \mathrm{~B}}$.

(A color version of this figure is available in the online journal.)

protostar (projected distance $\sim 8000 \mathrm{AU}$ ) is indistinguishable from that observed at the on-source positions (projected distance $\lesssim 2000 \mathrm{AU}$ ) of all the other sources in our sample, pointing to a common origin in both cases. The $\mathrm{CO}$ excitation at the outflow lobe is likely due to heating of the low-density $\left(n\left(\mathrm{H}_{2}\right) \lesssim 10^{6} \mathrm{~cm}^{-3}\right)$ gas in the outflow shocks and not from the protostellar accretion-generated PDRs.

The UV flux generated by protostellar accretion will heat up the dense molecular material close to the protostar and this material is likely to contribute to the $\mathrm{CO}$ emission. Indeed, the narrow velocity component $\left(\mathrm{FWHM} \lesssim 2 \mathrm{~km} \mathrm{~s}^{-1}\right.$ ) which originates in the UV-heated cavity walls is seen in the line profiles of the $\mathrm{CO} J=6-5$ and $\mathrm{CO} J=7-6$ transitions observed toward low-mass protostars (e.g., van Kempen et al. 2009a, 2009b; Yildiz et al. 2012). For these low- $J$ lines, this narrow component dominates the line flux. A similar narrow component is also seen in the $\mathrm{CO} J=10-9$ line for the protostars observed with Herschel/HIFI (Y1ld1z et al. 2010). However, in most cases the line flux is dominated by emission from the broad (FWHM = 25-30 $\mathrm{km} \mathrm{s}^{-1}$ ) component (Yıldız et al. 2010). Moreover, for the spectrally resolved lines, the broad component originating in shock-heated gas is seen to increase in strength going from $J_{\text {up }}=1$ to $J_{\text {up }}=10$ (Y1ldiz et al. 2010, 2012; van Kempen et al. $2009 b)$. Therefore, for the higher excitation CO $\left(J_{\text {up }} \geqslant 14\right)$ lines observed with PACS, emission from PDRs is unlikely to be the dominant contributor to the total flux.

\subsection{Origin in Outflow Shocks}

\subsubsection{Shocks along Envelope Cavity Walls}

Models for CO emission from shocks along envelope cavity walls predict most of the emission that contributes to the observed $\mathrm{CO}$ line fluxes originates in the high-density (preshock density $\gtrsim 10^{6} \mathrm{~cm}^{-3}$ ) gas (Visser et al. 2012). Our modeling here, however, suggests that the CO excitation is subthermal and that the observed emission likely originates in low-density gas $\left(n\left(\mathrm{H}_{2}\right) \lesssim 10^{6} \mathrm{~cm}^{-3}\right)$. Moreover, we find that the $\mathrm{CO}$ emission properties observed for the outflow position $\gtrsim 8000$ AU away from the protostar and for the on-source positions of the protostars in our sample are essentially indistinguishable. All these results suggest that the shocked gas producing $\mathrm{CO}$ emission is likely not located at the denser part of the cavity walls close to the protostar.

\subsubsection{CO Emission from Low-density Shock-heated Gas}

The optimal solutions obtained from both the isothermal and power-law temperature models indicate that the observed $\mathrm{CO}$ emission from protostars in our sample arises in low-density $\left(n\left(\mathrm{H}_{2}\right) \lesssim 10^{6} \mathrm{~cm}^{-3}\right)$ molecular gas which has high-temperature $(T>2000 \mathrm{~K})$ components. Such high temperatures and low densities are typical of gas heated by outflow shocks and have been reported by previous studies of FIR CO lines from protostars (Ceccarelli et al. 1998; Nisini et al. 1999; Giannini et al. 2001). The low densities inferred for the observed CO emission are more compatible with shock-heated gas within the envelope cavity along the molecular outflow or at envelope radius ¿several 100-1000 AU. Since the size of the central spaxel, from which the analyzed spectra are extracted, corresponds to a projected radius of $2000 \mathrm{AU}$ from the protostars, the shock emission must come from within this distance. Indeed, compact molecular outflows on $\lesssim 1000$ AU scales with high outflow momentum rates have been observed toward protostars (e.g., Santiago-García et al. 2009; Lee et al. 2009; Takahashi \& Ho 2012).

Non-dissociative shocks with shock speed $v_{s}>25 \mathrm{~km} \mathrm{~s}^{-1}$ can heat the gas to temperatures $>2000 \mathrm{~K}$ (Kaufman $\&$ Neufeld 1996). In general, outflows in protostars produce multiple shocks with varying velocities and different shock-front geometries. If the densities of the post-shock gas are low, e.g., $n\left(\mathrm{H}_{2}\right)<10^{4.5} \mathrm{~cm}^{-3}$, then the isothermal model predicts that the hottest $(T \gtrsim 2000 \mathrm{~K})$ gas component dominates the $\mathrm{CO}$ emission in all the transitions observed with PACS. Emitting gas at temperatures of 2000-5000 K can also produce the narrow range in the observed rotational temperatures. For slightly higher densities, e.g., $10^{5} \mathrm{~cm}^{-3} \lesssim n\left(\mathrm{H}_{2}\right) \lesssim 10^{6} \mathrm{~cm}^{-3}$, multiple gas components whose temperature follows a power-law distribution with index $b$ ranging from 2 to 3 can explain the observed FIR CO rotational diagrams. Even in this case, the hotter $(T \gtrsim 1000 \mathrm{~K})$ gas components produce most of the $\mathrm{CO}$ emission $\left(J_{\text {up }} \gtrsim\right.$ 18) observed with PACS. The post-shock gas in bow-shaped C-shocks can have a power-law temperature distribution (Smith et al. 1991; Neufeld \& Yuan 2008; Yuan \& Neufeld 2011; Giannini et al. 2011). Several such bow shocks are likely to be present along the supersonic molecular outflow from protostars even within a projected radius of 2000 AU. Classical bow shocks with a parabolic shape are expected to produce a power-law index, $b \sim 3.8$ (Smith \& Brand 1990; Neufeld et al. 2006; Neufeld \& Yuan 2008). Our results, however, indicate that $b$ is in the range of 2-3 for the observed FIR CO emission from protostars; a similar range for the power-law index have also been found for $\mathrm{H}_{2}$ emission from shocked gas (Neufeld et al. 2009; Yuan \& Neufeld 2011). As pointed out by Yuan \& Neufeld (2011), the lower values of $b$ suggest that either the curvature of the shock front is smaller than that of a parabola, or a mixture of shocks with different shock-front geometries ranging from planar to bow is present along the flow.

The observed outflow momentum rates $(\dot{M} v)$ in protostars are found to scale with $L_{\text {bol }}$ (Bontemps et al. 1996; Takahashi $\&$ Ho 2012). This correlation, which extends over three orders of magnitude in $L_{\mathrm{bol}}$, is primarily driven by the increase in the mass-loss rate in the outflows (Bontemps et al. 1996). There is also evidence that the mass-loss rate $(\dot{M})$ from young T Tauri stars and protostars scales with the accretion rate (Hartigan et al. 1995; I. S. Remming et al., in preparation). Since $L_{\text {bol }}$ increases with accretion luminosity in protostars, the mass-loss rates from outflows is expected to scale with $L_{\mathrm{bol}}$, and therefore 
the total mechanical luminosity in shocks should also scale with $L_{\text {bol }}$. Higher cooling rates are therefore expected for higher $L_{\text {bol }}$ sources, which, as Figure 10 shows, is tracked by $L_{\mathrm{CO}}$. Thus, shock heating of a lower density $\left(n\left(\mathrm{H}_{2}\right) \lesssim 10^{6} \mathrm{~cm}^{-3}\right)$ gas can at least qualitatively explain the observed trend between $L_{\mathrm{CO}}$ and $L_{\text {bol }}$ as well as the observed lack of dependence of $T_{\text {rot }}$ on $L_{\text {bol }}$ and $T_{\text {bol }}$.

\section{CONCLUSIONS}

We have analyzed the emission lines due to the rotational transitions of CO ( $J=14-13$ up to $J=46-45)$ in the FIR $(57-196 \mu \mathrm{m})$ spectra of 21 protostars in Orion, obtained with Herschel/PACS. The observed CO lines originate in the warm and hot gas within a projected distance of $\lesssim 2000$ AU from the protostars. We searched for correlations between the observed $\mathrm{CO}$ emission properties and protostellar luminosity, evolutionary status, and envelope density. We modeled the $\mathrm{CO}$ lines to constrain the excitation conditions in the emitting gas and identify possible heating mechanisms. Our main conclusions are listed below.

1. The total luminosity of the CO lines observed with PACS increases with protostellar luminosity over a large range $\left(2-217 L_{\odot}\right)$.

2. The CO rotational diagrams of protostars obtained with PACS show a positive curvature and the rotational temperature implied by the line ratios increases with increasing rotational quantum number $J$. A minimum of 3-4 rotational temperature components are required to fit the observed rotational diagram in the PACS wavelength range. The rotational temperature computed from the lowest- $J$ lines ( $J_{\text {up }}=$ 14-25) observed, $T_{\mathrm{R} 1}$, is found to lie within a narrow range for protostars while $L_{\text {bol }}$ values range over two orders of magnitude. A similar lack of dependence with $L_{\text {bol }}$ is also observed for the rotational temperatures determined for the higher- $J$ lines.

3. The observed $\mathrm{CO}$ emission properties $\left(L_{\mathrm{CO}}, T_{\text {rot }}\right)$ are uncorrelated with evolutionary indicators and envelope properties of the protostars such as $T_{\mathrm{bol}}$ and envelope density.
4. If the CO emitting gas is thermally excited, i.e., in LTE, multiple gas components at different temperatures are required to reproduce the observed emission. The temperatures of these components must somehow remain independent of protostellar luminosity over two orders of magnitude.

5. The observed CO emission can also be modeled as arising from sub-thermally excited gas with high-temperature $(T \gtrsim 2000 \mathrm{~K})$ components. An isothermal medium at uniform density can reproduce the observed emission for densities, $n\left(\mathrm{H}_{2}\right) \lesssim 10^{4.5} \mathrm{~cm}^{-3}$ and temperatures, $T \gtrsim 2000 \mathrm{~K}$. Observed emission can also be reproduced by a uniform density medium with a power-law temperature distribution $\left(T_{\min }=10 \mathrm{~K} \& T_{\max }=5000 \mathrm{~K}\right)$ for densities in the range of $10^{5}-10^{6} \mathrm{~cm}^{-3}$.

6. Emission from PDRs, produced along the UV-heated envelope cavity walls, is unlikely to be the dominant component of the $\mathrm{CO}$ emission observed in the PACS wavelength range. We argue that the simplest explanation for both the observed correlation between $L_{\mathrm{CO}}$ and $L_{\text {bol }}$ and the invariance of $\mathrm{CO}$ rotational temperatures between protostars is that the $\mathrm{CO}$ emission is dominated by sub-thermally excited, shock-heated gas at high temperatures $(T \gtrsim 2000 \mathrm{~K})$ and low densities $\left(n\left(\mathrm{H}_{2}\right) \lesssim 10^{6} \mathrm{~cm}^{-3}\right)$, located within the outflow cavities along the molecular outflow or along the cavity walls at radii $\gtrsim$ several 100-1000 AU.

\section{APPENDIX}

\section{SPECTRAL ENERGY DISTRIBUTIONS OF PROTOSTARS}

The observed SEDs of the protostars in our sample are shown in Figure 20. The $J, H$, and $K_{s}$ photometry, when available, is from the Two Micron All Sky Survey (2MASS; Skrutskie et al. 2006). Broadband fluxes at 3.6, 4.5, 5.8, 8.0, and $24.0 \mu \mathrm{m}$ were obtained in a joint survey of the Orion A and B molecular clouds by the Infrared Array Camera (IRAC; Fazio et al. 2004) and Multiband Imaging Photometer (MIPS; Rieke et al. 2004) aboard Spitzer (Kryukova et al. 2012; Megeath et al. 2012). Spitzer IRS (Houck et al. 2004) spectra also shown in Figure 20 were obtained as part of the GO programs 30859 and 50374
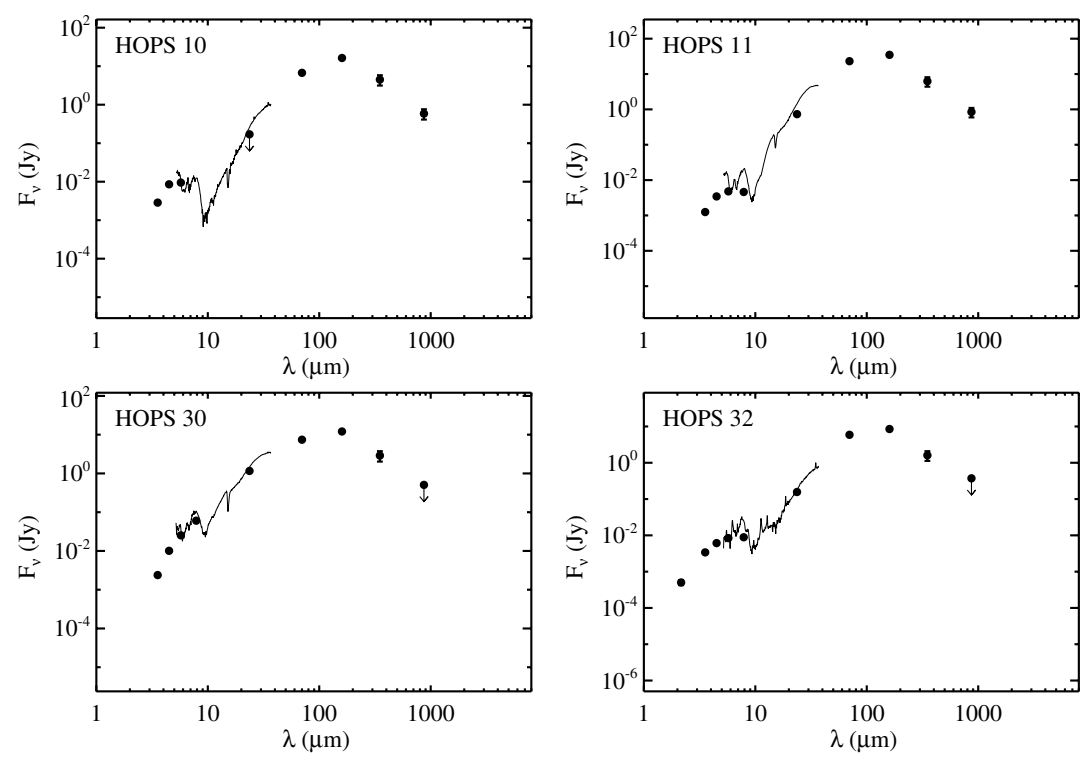

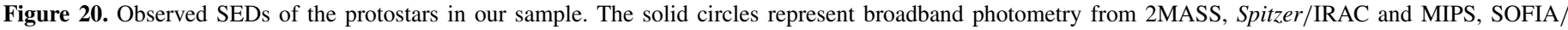

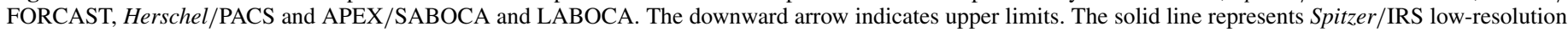
spectra. 

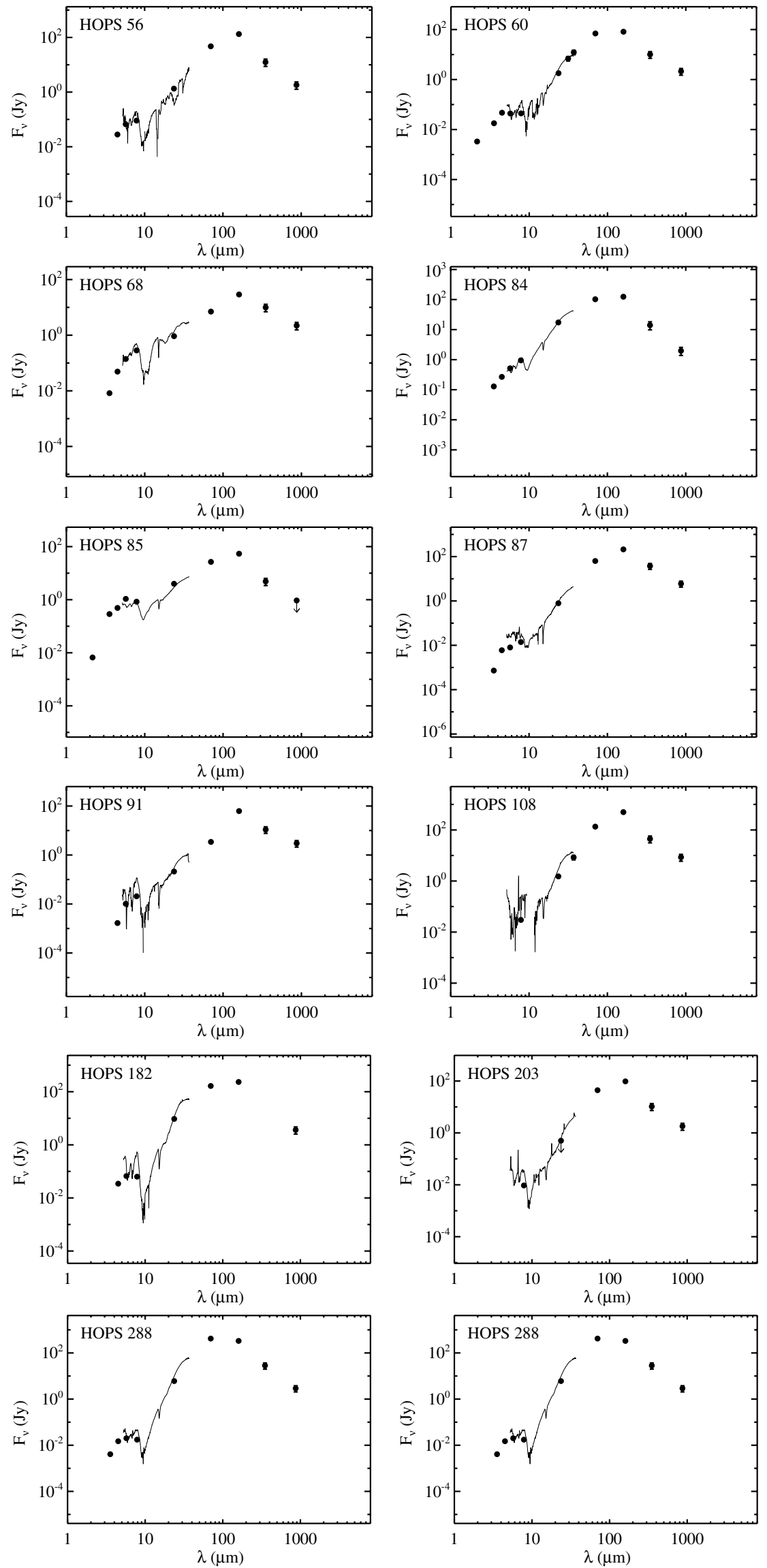

Figure 20. (Continued) 

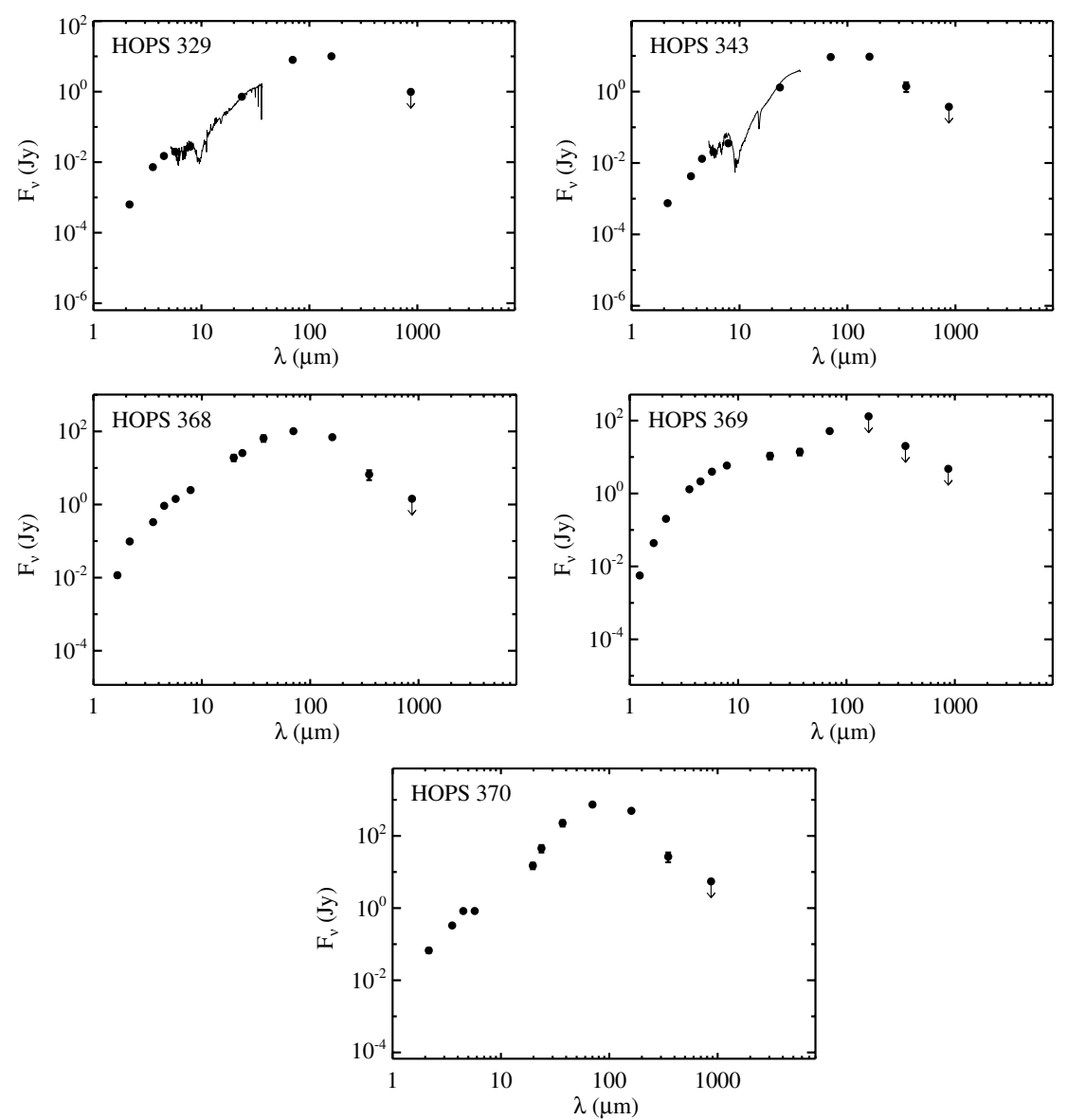

Figure 20. (Continued)

(PI: Megeath, S. T.; C. A. Poteet et al., in preparation; Poteet et al. 2011). For five sources toward the OMC-2 region (HOPS $60,108,368,369$, and 370), photometry at 19.7, 31.4, and $37.1 \mu \mathrm{m}$ was obtained with SOFIA/FORCAST (Adams et al. 2012). Images at 70 and $160 \mu \mathrm{m}$ of all the protostars in our sample were obtained with Herschel/PACS as part of the HOPS program (Fischer et al. 2010; Stanke et al. 2010; Fischer et al. 2012). Fluxes at these wavelengths were measured within an aperture of 9.'6 radius at $70 \mu \mathrm{m}$ and a 12 .". radius at $160 \mu \mathrm{m}$ after subtracting the median sky background estimated from an annulus extending from the aperture limit to twice that value (W. J. Fischer et al., in preparation). In addition, we also obtained submillimeter observations of our sample sources at $350 \mu \mathrm{m}$ with the Submillimeter APEX Bolometer Camera (SABOCA) and at $870 \mu \mathrm{m}$ with the Large APEX Bolometer Camera (LABOCA) on the Atacama Pathfinder Experiment (T. Stanke et al., in preparation). For the submillimeter data, we used the peak flux density per beam observed toward the source to construct the SEDs; the FWHMs of the beams were $7^{\prime \prime} .3$ at $350 \mu \mathrm{m}$ and $19^{\prime \prime}$ at $870 \mu \mathrm{m}$.

\section{REFERENCES}

Adams, J. D., Herter, T. L., Osorio, M., et al. 2012, ApJL, 749, 24

Ali, B., Tobin, J. J., Fischer, W. J., et al. 2010, A\&A, 518, L119

André, P., \& Montmerle, T. 1994, ApJ, 420, 837

André, P., Ward-Thompson, D., \& Barsony, M. 1993, ApJ, 406, 122

Bachiller, R. 1996, ARA\&A, 34, 111

Bachiller, R., \& Tafalla, M. 1999, in NATO ASIC Proc. 540: The Origin of Stars and Planetary Systems, ed. C. J. Lada \& N. D. Kylafis (Dordrecht: Kluwer), 227
Benedettini, M., Giannini, T., Nisini, B., et al. 2000, A\&A, 359, 148 Bontemps, S., André, P., Terebey, S., \& Cabrit, S. 1996, A\&A, 311, 858 Cassen, P., \& Moosman, A. 1981, Icarus, 48, 353

Ceccarelli, C. 2000, in ISO Beyond the Peaks: The 2nd ISO Workshop on Analytical Spectroscopy, ed. A. Salama, M. F. Kessler, K. Leech, \& B. Schulz (ESA Special Publication, Vol. 456; Noordwijk: ESA), 141 Ceccarelli, C., Caux, E., White, G. J., et al. 1998, A\&A, 331, 372 Chen, H., Myers, P. C., Ladd, E. F., \& Wood, D. O. S. 1995, ApJ, 445, 377

Chini, R., Reipurth, B., Ward-Thompson, D., et al. 1997, ApJL, 474, 135 Draine, B. T., Roberge, W. G., \& Dalgarno, A. 1983, ApJ, 264, 485

Enoch, M. L., Evans, N. J., Sargent, A. I., \& Glenn, J. 2009, ApJ, 692, 973

Evans, N. J., Dunham, M. M., Jørgensen, J. K., et al. 2009, ApJS, 181, 321

Fazio, G. G., Hora, J. L., Allen, L. E., et al. 2004, ApJS, 154, 10

Feigelson, E. D., \& Nelson, P. I. 1985, ApJ, 293, 192

Fich, M., Johnstone, D., van Kempen, T. A., et al. 2010, A\&A, 518, L86

Fischer, W. J., Megeath, S. T., Ali, B., et al. 2010, A\&A, 518, L122

Fischer, W. J., Megeath, S. T., Tobin, J. J., et al. 2012, ApJ, 756, 99

Giannini, T., Nisini, B., \& Lorenzetti, D. 2001, ApJ, 555, 40

Giannini, T., Nisini, B., Neufeld, D., et al. 2011, ApJ, 738, 80

Goicoechea, J. R., Cernicharo, J., Karska, A., et al. 2012, A\&A, 548, 77

Goldsmith, P. F., \& Langer, W. D. 1999, ApJ, 517, 209

Hartigan, P., Edwards, S., \& Ghandour, L. 1995, ApJ, 452, 736

Hartmann, L. (ed.) 2009, Accretion Processes in Star Formation (2nd ed.; Cambridge: Cambridge Univ. Press)

Herczeg, G. J., Karska, A., Bruderer, S., et al. 2012, A\&A, 540, A84

Hollenbach, D., \& McKee, C. F. 1989, ApJ, 342, 306

Houck, J. R., Roellig, T. L., van Cleve, J., et al. 2004, ApJS, 154, 18

Isobe, T., Feigelson, E. D., \& Nelson, P. I. 1986, ApJ, 306, 490

Jaffe, D. T., Harris, A. I., \& Genzel, R. 1987, ApJ, 316, 231

Kaufman, M. J., \& Neufeld, D. A. 1996, ApJ, 456, 611

Kenyon, S. J., Calvet, N., \& Hartmann, L. 1993, ApJ, 414, 676

Kenyon, S. J., \& Hartmann, L. 1995, ApJS, 101, 117

Kryukova, E., Megeath, S. T., Gutermuth, R. A., et al. 2012, AJ, 144, 31 
Lada, C. J. 1987, in IAU Symp. 115, Star Forming Regions, ed. M. Peimbert \& J. Jugaku (Cambridge: Cambridge Univ. Press), 1

Lavalley, M., Isobe, T., \& Feigelson, E. 1992, in ASP Conf. Ser. 25, Astronomical Data Analysis Software and Systems I, ed. D. M. Worrall, C. Biemesderfer, \& J. Barnes (San Francisco, CA: ASP), 245

Lee, C.-F., Hirano, N., Palau, A., et al. 2009, ApJ, 699, 1584

McKee, C. F., \& Ostriker, E. C. 2007, ARA\&A, 45, 565

Megeath, S. T., Gutermuth, R., Muzerolle, J., et al. 2012, AJ, 144, 192

Myers, P. C., \& Ladd, E. F. 1993, ApJL, 413, 47

Neufeld, D. A. 2012, ApJ, 749, 125

Neufeld, D. A., \& Hollenbach, D. J. 1994, ApJ, 428, 170

Neufeld, D. A., Melnick, G. J., Sonnentrucker, P., et al. 2006, ApJ, 649, 816

Neufeld, D. A., Nisini, B., Giannini, T., et al. 2009, ApJ, 706, 170

Neufeld, D. A., \& Yuan, Y. 2008, ApJ, 678, 974

Nisini, B., Benedettini, M., Giannini, T., et al. 1999, A\&A, 350, 529

Nisini, B., Giannini, T., \& Lorenzetti, D. 2002, ApJ, 574, 246

Nisini, B., Saraceno, P., Ceccarelli, C., et al. 1997, in The Far Infrared and Submillimetre Universe., ed. A. Wilson (ESA Special Publication, Vol. 401; Noordwijk: ESA), 321

Ott, S. 2010, in ASP Conf. Ser. 434, Astronomical Data Analysis Software and Systems XIX, ed. Y. Mizumoto, K.-I. Morita, \& M. Ohishi (San Francisco, CA: ASP), 139

Pilbratt, G. L., Riedinger, J. R., Passvogel, T., et al. 2010, A\&A, 518, L1

Poglitsch, A., Waelkens, C., Geis, N., et al. 2010, A\&A, 518, L2

Poteet, C. A., Megeath, S. T., Watson, D. M., et al. 2011, ApJL, 733, 32

Reipurth, B., \& Bally, J. 2001, ARA\&A, 39, 403

Rieke, G. H., Young, E. T., Engelbracht, C. W., et al. 2004, ApJS, 154, 25

Santiago-García, J., Tafalla, M., Johnstone, D., \& Bachiller, R. 2009, A\&A, 495, 169

Saraceno, P., Benedettini, M., di Giorgio, A. M., et al. 1999a, in The Physics and Chemistry of the Interstellar Medium, ed. V. Ossenkopf, J. Stutzki, \& G. Winnewisser (Herdecke: GCA-Verlag), 279

Saraceno, P., Nisini, B., Benedettini, M., et al. 1999b, in The Universe as Seen by ISO, ed. P. Cox \& M. Kessler (ESA Special Publication, Vol. 427; Noordwijk: ESA), 575

Shu, F. H., Adams, F. C., \& Lizano, S. 1987, ARA\&A, 25, 23

Skrutskie, M. F., Cutri, R. M., Stiening, R., et al. 2006, AJ, 131, 1163

Smith, M. D., \& Brand, P. W. J. L. 1990, MNRAS, 245, 108

Smith, M. D., Brand, P. W. J. L., \& Moorhouse, A. 1991, MNRAS, 248, 451
Spaans, M., Hogerheijde, M. R., Mundy, L. G., \& van Dishoeck, E. F. 1995, ApJL, 455, 167

Stacey, G. J., Kurtz, N. T., Smyers, S. D., \& Harwit, M. 1983, MNRAS, 202, $25 \mathrm{P}$

Stacey, G. J., Kurtz, N. T., Smyers, S. D., et al. 1982, ApJL, 257, 37

Stahler, S. W., \& Palla, F. (ed.) 2005, The Formation of Stars (New York: Wiley)

Stanke, T., Stutz, A. M., Tobin, J. J., et al. 2010, A\&A, 518, L94

Storey, J. W. V., Watson, D. M., Townes, C. H., Haller, E. E., \& Hansen, W. L. 1981, ApJ, 247, 136

Takahashi, S., \& Ho, P. T. P. 2012, ApJL, 745, 10

Takahashi, S., Saito, M., Ohashi, N., et al. 2008, ApJ, 688, 344

Terebey, S., Shu, F. H., \& Cassen, P. 1984, ApJ, 286, 529

Tobin, J. J., Hartmann, L., Calvet, N., \& D’Alessio, P. 2008, ApJ, 679, 1364

van Dishoeck, E. F. 2004, ARA\&A, 42, 119

van Dishoeck, E. F., van Kempen, T. A., \& Güsten, R. 2009, in ASP Conf. Ser. 417, Submillimeter Astrophysics and Technology: A Symposium Honoring Thomas G. Phillips, ed. D. C. Lis, J. E. Vaillancourt, P. F. Goldsmith, T. A. Bell, N. Z. Scoville, \& J. Zmuidzinas (San Francisco, CA: ASP), 203

van Kempen, T. A., Green, J. D., Evans, N. J., et al. 2010a, A\&A, 518, L128

van Kempen, T. A., Kristensen, L. E., Herczeg, G. J., et al. 2010b, A\&A, 518, L121

van Kempen, T. A., van Dishoeck, E. F., Güsten, R., et al. 2009a, A\&A, 507,1425

van Kempen, T. A., van Dishoeck, E. F., Güsten, R., et al. 2009b, A\&A, 501,633

Visser, R., Kristensen, L. E., Bruderer, S., et al. 2012, A\&A, 537, A55

Watson, D. M., Genzel, R., Townes, C. H., \& Storey, J. W. V. 1985, ApJ, 298,316

Watson, D. M., Storey, J. W. V., Townes, C. H., Haller, E. E., \& Hansen, W. L. 1980, ApJL, 239, 129

Whitney, B. A., Wood, K., Bjorkman, J. E., \& Wolff, M. J. 2003, ApJ, 591, 1049

Wilking, B. A. 1989, PASP, 101, 229

Williams, J. P., Plambeck, R. L., \& Heyer, M. H. 2003, ApJ, 591, 1025

Yang, B., Stancil, P. C., Balakrishnan, N., \& Forrey, R. C. 2010, ApJ, 718, 1062 Yıldız, U. A., Kristensen, L. E., van Dishoeck, E. F., et al. 2012, A\&A, 542, A86

Yıldız, U. A., van Dishoeck, E. F., Kristensen, L. E., et al. 2010, A\&A, 521, L40

Yuan, Y., \& Neufeld, D. A. 2011, ApJ, 726, 76 